\title{
Measurement of the Noise Resulting from the Interaction of Turbulence with a Lifting Surface
}

\author{
Florence V. Hutcheson ${ }^{1}$, Thomas F. Brooks ${ }^{2}$, Casey L. Burley ${ }^{3}$ \\ Aeroacoustics Branch \\ Daniel J. Stead ${ }^{4}$ \\ Lockheed Martin Engineering and Sciences \\ NASA Langley Research Center \\ Hampton, Virginia 23681-2199
}

\begin{abstract}
An experimental study of the noise resulting from the interaction of an airfoil with incident turbulence is presented. The test models include NACA0015 airfoils of different chord lengths, a flat plate with a sharp leading edge, and an airfoil of same section as a reference Fowler flap. The airfoils are immersed in nearly isotropic turbulence. Two approaches for performing the noise measurements are used and compared. The effects that turbulence intensity and scales, airfoil geometry, velocity and angle of attack have on the incident turbulence interaction noise are examined. Detailed directivity measurements are presented. It is found that noise spectral levels beyond the peak frequency decrease more with decreasing airfoil leading edge sharpness, and that spectral peak level (at $0^{\circ}$ angle of attack) appears to be mostly controlled by the airfoil's thickness and chord. Increase in turbulence scale and intensity are observed to lead to a uniform increase of the noise spectral levels with an $L I^{2}$ dependence (where $L$ is the turbulence longitudinal integral scale and $I$ is the turbulence intensity). Noise levels are found to scale with the $6^{\text {th }}$ power of velocity and the $2^{\text {nd }}$ power of the airfoil chord. Sensitivity to changes in angle of attack appears to have a turbulence longitudinal integral scale to chord $(C)$ ratio dependence, with large effects on noise for $\mathrm{L} / \mathrm{C} \geq 1$ and decreased effects as $\mathrm{L} / \mathrm{C}$ becomes smaller than 1 . For all $\mathrm{L} / \mathrm{C}$ values, the directivity pattern of the noise resulting from the incident turbulence is seen to remain symmetric with respect to the direction of the mean flow until stall, at which point, the directivity becomes symmetric with respect to the airfoil chord. It is also observed that sensitivity to angle of attack changes is more pronounced on the model suction side than on the model pressure side, and in the higher frequency range of the spectra for the largest airfoils tested $(\mathrm{L} / \mathrm{C}<0.24)$.
\end{abstract}

\section{Introduction}

A number of numerical [Ref. 1-6] and experimental [Ref. 7-12] studies have been conducted in order to characterize and model the noise resulting from the interaction of turbulent flow with a lifting surface. This interaction occurs, for example, during aircraft takeoff and landing when the landing gear wake impinges onto a flap, or with helicopter rotor blades as an advancing blade encounters the wakes from preceding blades.

A benchmark theoretical and experimental study was conducted by Paterson and Amiet [2] in the 1970s. They measured the noise and unsteady surface pressure from a 9" chord NACA0012 airfoil in homogeneous turbulence. For the flow speeds tested, the turbulence longitudinal integral scale was approximately $15 \%$ of the airfoil's chord and the turbulence intensity was around $4 \%$. The flow Mach number ranged from 0.1 to 0.5 . Far field noise spectra and directivity were acquired. Paterson and Amiet reported that at $0^{\circ}$ angle of attack (focus of their study), the noise directivity followed that of a compact

\footnotetext{
${ }^{1}$ Research Scientist, AIAA member

${ }^{2}$ Senior Research Scientist, AIAA Fellow

${ }^{3}$ Senior Research Scientist, AIAA member

${ }^{4}$ Senior Research Engineer
} 
dipole at low frequencies and that of a baffled dipole (edge radiation from a semi-infinite plate) at higher frequencies. They also reported that varying the airfoil effective angle of attack between $0^{\circ}$ and $6.3^{\circ}$ had little effect ( 1 to $2 \mathrm{~dB}$ ) on noise levels for all velocities and on the directivity which was only obtained on the pressure side of the airfoil. They predicted the noise and surface pressure from measured turbulence statistics and thin airfoil theory, and found good agreement with the measured data over the lower frequency range.

In more recent studies, Moreau et al. [11] performed incident turbulence noise measurements on a 4" chord NACA0012 and flat plate as well as on a 5.4" chord cambered airfoil designed for use in automotive engine cooling fans. The turbulence integral scales were approximately $9 \%$ of the chord of the flat plate and NACA0012, and 6.5\% of chord of the cambered airfoil. The relative thicknesses of the flat plate and cambered airfoil were 0.12 " and 0.1 ", respectively. The flow speeds ranged from $20 \mathrm{~m} / \mathrm{s}$ to $40 \mathrm{~m} / \mathrm{s}$ (corresponding to Mach numbers around 0.1) and the angle of attack was varied between $0^{\circ}$ and $15^{\circ}$ (estimated to correspond to effective angles of attack of $0^{\circ}$ and $5.4^{\circ}$ due to the open jet set-up [12]). Directivity measurements were limited to aft angles between $105^{\circ}$ and $160^{\circ}$ (as measured from the upstream axis) on either side of the test models. Moreau et al. observed that no significant change in noise level and directivity pattern occurred with angle of attack changes, that the noise levels scaled best with fifth power of velocity, and that the thickest airfoil tested was quieter than the other airfoils in the higher frequency range (as first observed by Olsen [19]). Moreau et al. also extended Amiet's original noise prediction model [1] that is valid for parallel gusts on flat plates, to 3-dimensional supercritical and subcritical gusts. Their prediction model was found to agree well with the experimental data obtained with the thinnest airfoils where thin airfoil theory holds. Semi-empirical corrections were proposed to account for thickness effects.

Oelermans and Migliore [9] performed inflow turbulence noise measurements on a series of 9" chord airfoils designed for use on small wind turbines. The turbulence intensity was $11 \%$ at the airfoil leading edge location. The turbulence scales to airfoil chord or thickness ratios were not specified. The measurements were performed at a single location with a microphone array positioned close to the test section and centered at the mid-span of the airfoils trailing edge. For the data reported, the flow Mach number ranged between 0.06 to 0.18 and the effective angle of attack varied between 0 and $7.9^{\circ}$. Data was acquired from the suction side and pressure side of the models by modifying the airfoil's placement. The authors observed a high frequency increase in leading edge noise for high angles of attack and a sixth power velocity dependence of the noise levels. The difference in the spectral levels obtained from the pressure and suction sides of the airfoil placed at $7.9^{\circ}$ angle of attack was attributed to the $\sin ^{2}(\theta / 2)$ directivity ( $\theta$ being the emission angle) of a baffled dipole [2], indicating a symmetric directivity with respect to the chord. A comparison of the different airfoils' leading edge noise spectra showed a clear increase in noise with airfoil leading edge sharpness. Although the authors were able to acquire the incident turbulence noise data over a broad frequency range, and observe several trends for the high frequency part of the noise spectra, the measurements were limited to above $1 \mathrm{kHz}$, possibly missing the noise spectral peak.

The most recent study was conducted by Staubs and Devenport [12]. Their experiment encompassed a broad range of longitudinal integral scale to chord ratios as well as leading edge radius and airfoil thickness to integral scale ratios. They tested 5 airfoils, a 7.9" chord NACA0012, a 24" chord NACA0015 and NACA0012, and two 35.8" chord cambered airfoils (designed for wind turbines). The grid generated turbulence had an intensity of $3.9 \%$ and a longitudinal integral scale of 3.23". However, background noise from the turbulence grid limited the leading edge noise measurements to less than $1 \mathrm{kHz}$ for the thinnest airfoil tested and to less than $350 \mathrm{~Hz}$ for the thickest airfoil tested, bounding the analysis in part to the spectral peak region. Tones from the grid bars also affected the data in that low frequency range. Noise spectra were obtained for a single flow speed and from the average signal of a small group of microphones at a fixed location centered $90^{\circ}$ from the tunnel axis. Spectra were obtained only on the airfoil's suction side except for the smallest NACA0012 model for which pressure side measurements were also acquired. The authors also observed a reduction in noise in the higher frequency range for the thicker airfoils and an increase in noise of approximately $1 \mathrm{~dB}$ at the larger angles of attack tested.

In the present study, detailed directivity measurements of noise from incident turbulence were obtained on a series of NACA0015 airfoils to examine in a more systematic way the effect of airfoil thickness and chord on the radiated noise. The effects of changes in turbulence intensity, turbulence scales, freestream velocity and angle of attack on noise level were examined. Effects of changes in angle of attack on noise directivity (which were not fully examined in previous studies) are also a focus of this paper. This work is 
part of a larger study intended to establish a set of turbulence, surface pressure, and acoustic data for isolated airfoils immersed in turbulent flow. The data is to be used to improve the capabilities of existing prediction models such as those of a current rotorcraft blade-wake interaction noise model [Ref. 13]. This study also represents an initial step towards characterizing and modeling landing gear wake-flap interaction noise that occurs in a more complex geometrical setup. This paper focuses on the analysis of the acoustic data acquired. The effects that turbulence intensity and scales, airfoil geometry, velocity and angle of attack have on the resulting noise are examined. A detailed description of the approach used to perform the noise measurement is given.

\section{Test Description}

This experimental study was performed at NASA Langley Research Center in the Quiet Flow Facility (QFF). The QFF (shown in Figure 1) is specifically designed for anechoic testing and is equipped with a 2 by 3 foot rectangular open jet nozzle.

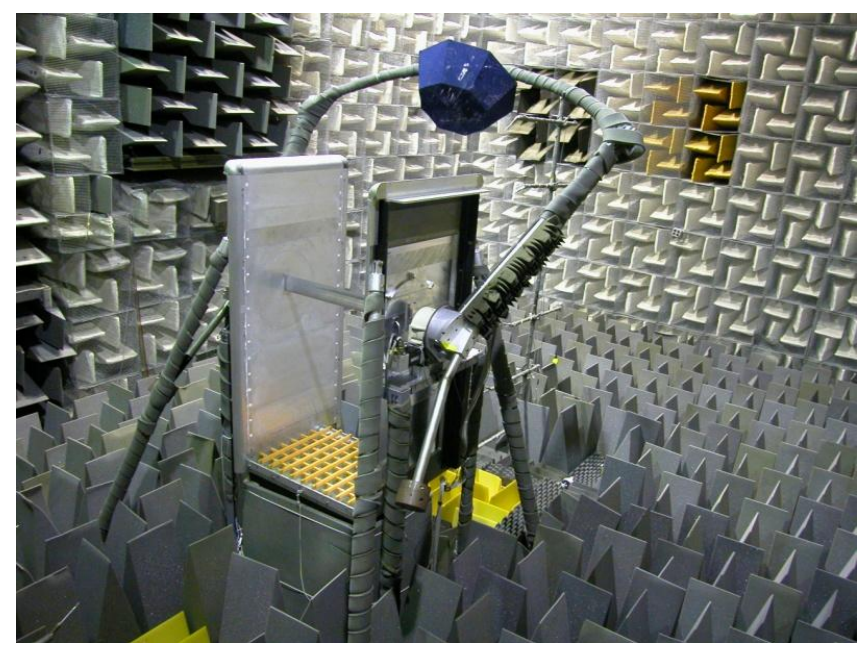

Figure 1. Quiet Flow Facility with turbulence grid and airfoil positioned in the test section.

\section{A. Turbulence grids}

The turbulence was generated by a grid mounted across the exit plane of the nozzle (as seen in Figure 1). Two different turbulence grids, grid A (shown in Figure 2) and grid B, were tested. Both had an open area ratio of $60 \%$. The bars of grid A were 0.5 " $\mathrm{x} 0.5$ " in cross section, and the spacing (center to center) between consecutive bars was 2.22 ". For grid B, the bars were 0.8 "x 0.8 " in cross section and the spacing (center to center) between consecutive bars was 3.55". Acoustic foam treatment was glued along the downstream side of each bar of the turbulence grids to attenuate noise from the air flowing through the grids.
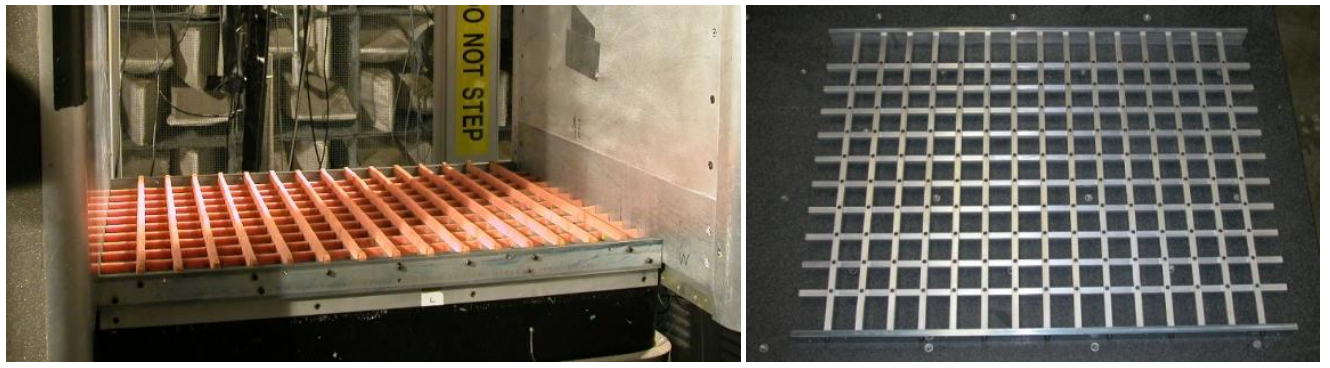

Figure 2. Turbulence grid A with and without acoustic foam treatment. 


\section{B. Test models}

Six different airfoils and one flat plate, all 3 feet in span, were tested. Five of the airfoils were NACA0015 of 1", 2", 4", 6" and 9" chord, respectively. The sixth airfoil configuration had the same airfoil section as a reference Fowler flap and a 4.8" chord. The plate had a 4" chord and sharp leading and trailing edges. Sketches of the test models' cross-sections are shown in Figure 3. Each test model was mounted with the leading edge (LE) positioned 41.5" downstream of the nozzle exit plane, in a region of the flow where the generated turbulence is expected (based on measurements) to be homogeneous and nearly isotropic. The models were supported above the turbulence grid by two vertical side plates that are mounted to the short sides of the nozzle. The models were tested tripped and un-tripped to examine noise sensitivity to the nominal laminar to turbulence transition location. For the tripped configurations, \#70 grit was applied over the first $20 \%$ chord on the upper and lower surfaces.

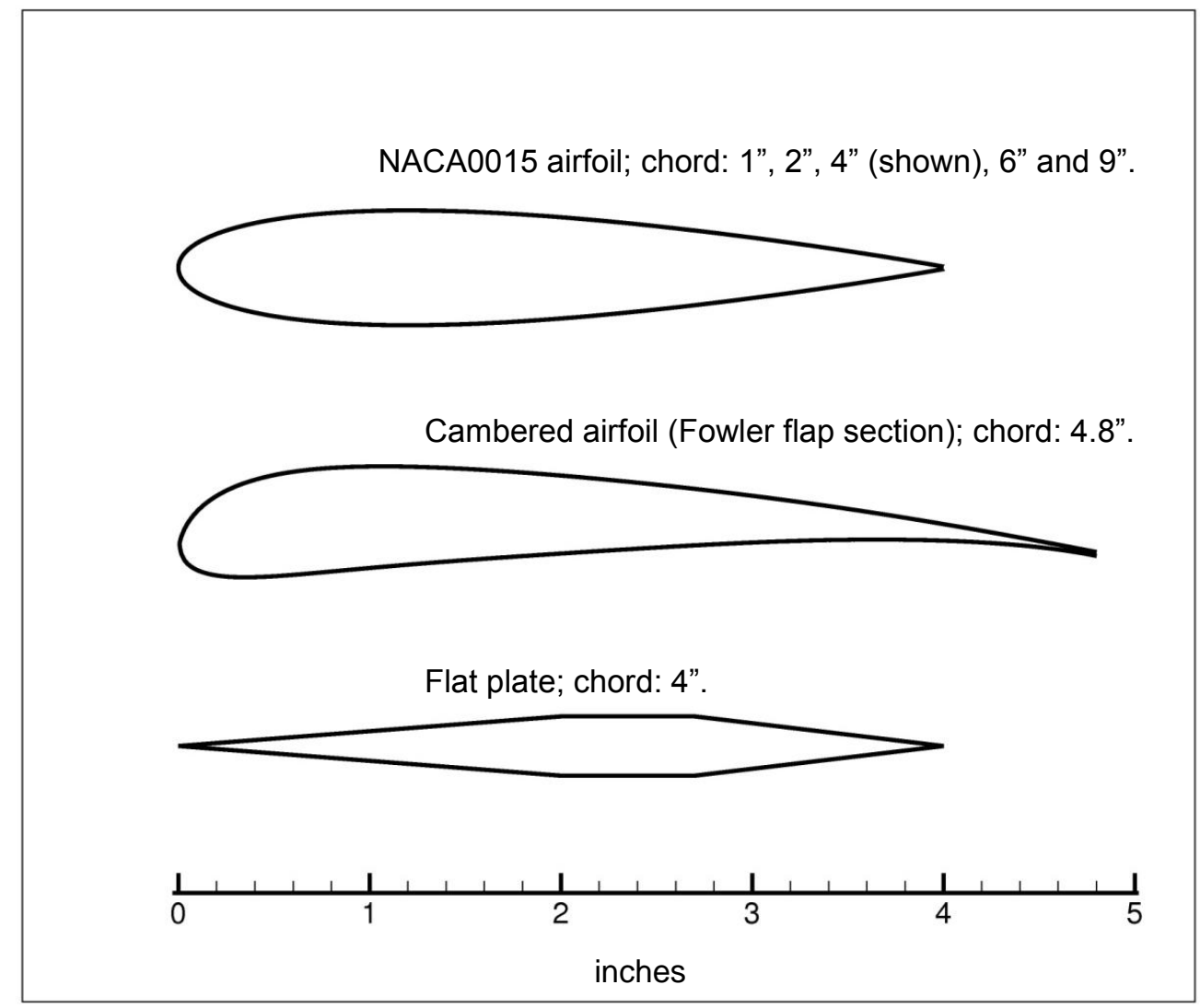

Figure 3. Test models cross-section.

\section{Microphones set-up}

Noise measurements were acquired for flow speed Mach numbers 0.09, 0.11 and 0.127 (maximum flow speed that could be reached with the nozzle grids in place) and for angles of attack $\alpha$ ranging from $0^{\circ}$ to $20^{\circ}$. Measurements for the flat plate model were performed only at $0^{\circ}$ angle of attack. A sketch of the acoustic test set-up is shown in Figure 4. Thirteen microphones were positioned on opposite sides of the test section, in the mid-span plane of the test airfoils. These microphones were directed at a point located along the nozzle centerline, 41.5 " above the nozzle exit plane (location of the airfoils' leading edge at midspan). The thirteen microphones were positioned $6.5 \mathrm{ft}$. away from that center point and at, respectively, $\Phi=-135^{\circ},-120^{\circ},-105^{\circ},-90^{\circ},-75^{\circ},-60^{\circ},-45^{\circ}, 135^{\circ}, 120^{\circ}, 105^{\circ}, 90^{\circ}, 75^{\circ}$ and $60^{\circ}$ elevation (as measured from the downstream direction). For practicality, noise measurements at $45^{\circ}$ elevation were acquired using one of the microphones of the Medium Aperture Directional Array (MADA). The MADA was positioned 5 
feet from the test models' leading edge. Acoustic measurements using the phased microphone array were also performed at $\pm 90^{\circ}$ and $\pm 45^{\circ}$ elevation angles for a limited number of airfoil configurations.

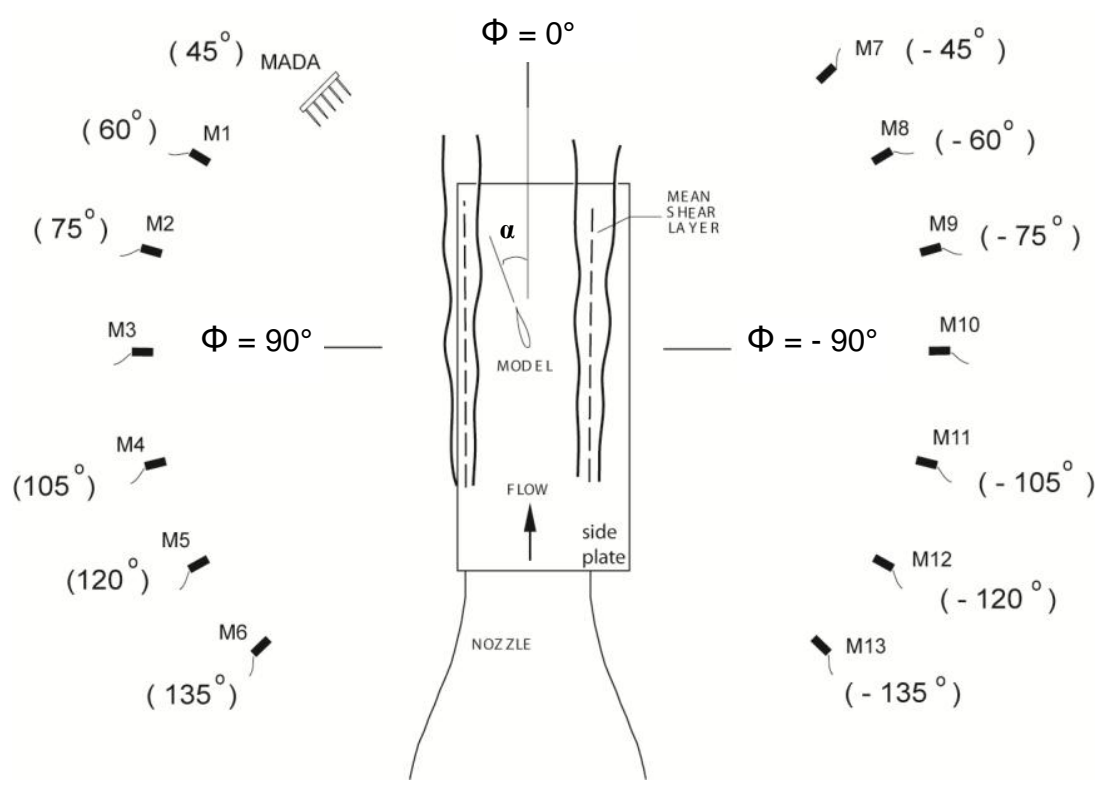

Figure 4. Acoustic test set up.

\section{Data acquisition}

Transient data recorders controlled by a workstation were used to acquire the data simultaneously from all the microphone channels at a sampling rate of $71.428 \mathrm{kHz}$. A low pass filter set at $25 \mathrm{kHz}$ was used to condition the outputs from each microphone channel. The microphone signals were also high pass filtered at $50 \mathrm{~Hz}$. A cross-spectral matrix was constructed for each set of data acquired from all the microphone channels (diagonal elements of the cross-spectral matrix representing the auto-spectra). Individual elements of the cross-spectral matrices were computed by partitioning each time signal into non-overlapping segments of $2^{12}$ samples. Each time history segment was then Fourier transformed using a Hamming window for signal conditioning. The resulting frequency resolution was $17.42 \mathrm{~Hz}$. This cross-spectral representation of the acquired acoustic signals is used subsequently for the analysis of the data.

\section{E. Turbulence characteristics}

Two-component hot-wire surveys were performed for each grid in the empty test section to measure turbulence intensities as well as wavenumber spectra and correlation functions at the streamwise station of the test models leading edge. Over the center portion (18"x12" area) of the survey plane, away from the test section side plates or from the jet shear layer, the wavenumber spectra and the turbulence intensities were found to be nearly constant which is consistent with homogeneous turbulence. The intensities calculated for the streamwise and transverse turbulence components were also found to be nearly equal. The values of the turbulence intensities (averaged over the center portion of the survey plane) for the three flow Mach numbers tested are listed in Table 1 for each grid. The longitudinal (streamwise) integral scales, L, inferred from the longitudinal wavenumber spectra and autocorrelation functions were 1.22" and 1.2", respectively, for grid A; and 1.63" and 1.29", respectively, for grid B. These integral scales were found to be relatively independent of tunnel speed. Average values (between the 2 methods of calculation) of 1.21" (for grid A) and 1.46" (for grid B) were chosen to represent the longitudinal integral scales for all of the experimental data. Finally, the ratio of the transverse to longitudinal integral scale was found to vary between 0.5 and 0.55 for grid $\mathrm{A}$, and between 0.55 and 0.58 for grid B depending on whether the integral scales were 
inferred from the wavenumber spectra or autocorrelation functions. This finding is consistent with definitions for isotropic turbulence. More detailed results of the turbulence measurements are to be provided in a follow on paper.

Table 1. Turbulence characteristics

\begin{tabular}{|l|c|c|c|c|c|c|}
\hline & \multicolumn{3}{|c|}{ Grid A } & \multicolumn{3}{c|}{ Grid B } \\
\hline Flow Mach \# & 0.09 & 0.11 & 0.127 & 0.09 & 0.11 & 0.127 \\
\hline Turbulence intensity, I $=<u^{\prime}>/ \mathrm{U}_{\infty}$ & $5.7 \%$ & $5.6 \%$ & $5.7 \%$ & $7.4 \%$ & $7.5 \%$ & $7.5 \%$ \\
\hline Longitudinal integral scale, $\mathrm{L}$ & \multicolumn{3}{|c|}{$1.21{ }^{\prime \prime}$} & \multicolumn{3}{c|}{$1.46 "$} \\
\hline
\end{tabular}

The resulting ratio of airfoil chord and thickness to the turbulence longitudinal integral scale are listed in Table 2 for each model tested.

Table 2. Test models characteristics

\begin{tabular}{|c|c|c|c|c|c|c|c|c|c|}
\hline \multirow[t]{2}{*}{ Test model } & \multirow{2}{*}{$\begin{array}{l}\text { Chord, } \\
\text { C }\end{array}$} & \multirow{2}{*}{$\begin{array}{c}\text { maximum } \\
\text { Thickness, } \\
1\end{array}$} & \multirow{2}{*}{$\begin{array}{l}\text { Leading edge } \\
\text { radius, } \mathrm{r}_{\mathrm{LE}}\end{array}$} & \multicolumn{2}{|c|}{$\mathrm{L} / \mathrm{C}$} & \multicolumn{2}{|c|}{$\mathrm{L} / \mathrm{l}$} & \multicolumn{2}{|c|}{$\mathrm{L} / \mathrm{r}_{\mathrm{LE}}$} \\
\hline & & & & grid: A & B & A & B & A & B \\
\hline NACA0015 & $1 "$ & $0.15 "$ & $0.024 "$ & 1.21 & 1.46 & 8.0 & 9.7 & 50.4 & 60.8 \\
\hline NACA0015 & 2" & $0.3 "$ & $0.049 "$ & 0.60 & 0.73 & 4.0 & 4.8 & 24.7 & 29.8 \\
\hline NACA0015 & 4" & $0.6 "$ & $0.099 "$ & 0.30 & 0.36 & 2.0 & 2.4 & 12.2 & 14.7 \\
\hline NACA0015 & 6" & $0.9 "$ & $0.149 "$ & 0.20 & 0.24 & 1.3 & 1.6 & 8.1 & 9.8 \\
\hline NACA0015 & 9" & $1.35^{\prime \prime}$ & $0.223 "$ & 0.13 & 0.16 & 0.9 & 1.0 & 5.4 & 6.5 \\
\hline $\begin{array}{l}\text { Cambered } \\
\text { airfoil }\end{array}$ & $4.8 "$ & $0.54 "$ & $\sim 0.15^{\prime \prime}$ & 0.25 & 0.30 & 2.2 & 2.9 & 6.0 & 7.3 \\
\hline Flat plate & 4" & $0.3 "$ & $\begin{array}{l}\text { Sharp LE, } \\
0.005 \text { " thick }\end{array}$ & 0.30 & 0.36 & 4.0 & 4.8 & - & - \\
\hline
\end{tabular}

\section{Noise measurement methodology}

Two approaches were used to obtain the spectra of the noise resulting from the model interaction with inflow turbulence. The first one is based on a Coherent Output Power (COP) method developed by Brooks and Hodgson [Ref. 15] (originally for the measurement of trailing edge noise). The COP method is consistent with the general coherent output power definition given by Bendat and Piersol [Ref. 16]. This noise measurement approach is based on the conceptual model that incident turbulence interaction noise is an edge pressure scattering phenomenon that has a dipole character, where the axis of the dipole is perpendicular to the mean flow and to the leading edge of the model. This concept is widely accepted for airfoils at $0^{\circ}$ angle of attack. In the present study this conceptual model is extended to non-zero angles of attack by assuming that the dipole axis remains perpendicular to the flow and not to the airfoil's chord line. Thus, it is assumed that the incident turbulence interaction noise spectrum $S(f)$ that would be measurable and coherent at the pair of microphones M3 and M10 (which referring to Figure 4 are located at $\Phi= \pm 90^{\circ}$ ) can be obtained from the cross spectrum $\mathrm{G}_{\mathrm{M} 3, \mathrm{M} 10}$ between the two microphone signals. The interaction noise at other microphone locations, $\mathrm{Mj}$, can then be obtained from

$$
\mathrm{S}_{\mathrm{COP}}(\mathrm{f})_{\text {at } \mathrm{Mj}}=\left|\mathrm{G}_{\mathrm{M} 3, \mathrm{Mj}}(\mathrm{f})\right|^{2} /\left|\mathrm{G}_{\mathrm{M} 3, \mathrm{M} 10}(\mathrm{f})\right|=\left|\mathrm{G}_{\mathrm{M} 10, \mathrm{Mj}}(\mathrm{f})\right|^{2} /\left|\mathrm{G}_{\mathrm{M} 3, \mathrm{M} 10}(\mathrm{f})\right|
$$

This cross-spectral approach has the advantage that only correlated noise is retained. Extraneous mutually incoherent noises received by microphones M3 and M10 are excluded. Possible contamination from other coherent noise sources (such as from the test rig, the junction of the airfoil side edges with the supporting side plates, or the airfoil trailing edge) however remains. Potential contributions from these extraneous sources are examined in following sections.

In the second approach, noise spectra at each microphone location are obtained directly from the microphone signals auto-spectra.

$$
S_{\text {auto }}(f)_{\text {at } M j}=\left|G_{M j, M j}(f)\right|
$$


This approach assumes that the noise resulting from the interaction of the airfoil with the incident turbulence dominates all other sources.

Note that for both methods, (as will be explained in the next section) the auto-spectra and cross-spectra obtained without the airfoil models in the test section are subtracted (on a pressure-squared basis) from the auto and cross-spectra obtained with the airfoil in place.

\section{A. Background noise}

\section{Noise Measurements Analysis and Results}

Noise from the empty test section was evaluated for each turbulence grid and flow speed. The noise spectra obtained (from microphone M10) at the highest flow speed are shown in Figure 5(a) and (b) along with the spectra obtained when the test models were installed in the test section at $0^{\circ}$ angle of attack (quietest turbulence/airfoil interaction noise configuration). It is seen that the turbulence grid treatment successfully prevented the formation of Aeolian tones from the grid bars and kept the background noise to an acceptable level. With turbulence grid A, noise from the test models was measureable up to 1 or 1.2 $\mathrm{kHz}$, while with grid $\mathrm{B}$, noise from most of the test models could be measured up to approximately 1.5 $\mathrm{kHz}$. In both cases, noise from the 1" chord airfoil was closest to the background level. However, at larger angles of attack, noise from the 1" airfoil increased significantly above background.

In the rest of this analysis, the background noise pressure field (measured with the turbulence flowing through the empty test section for each grid and flow speed) was subtracted from the noise pressure field measurements obtained with the different airfoil configurations. The spectra obtained after background noise subtraction for the previous test cases are shown in Figure 6(a) and (b). The spectra are truncated at upper frequencies where background noise significantly affected the spectra, and at frequencies below $140 \mathrm{~Hz}$ which is the anechoic cutoff of the test chamber.
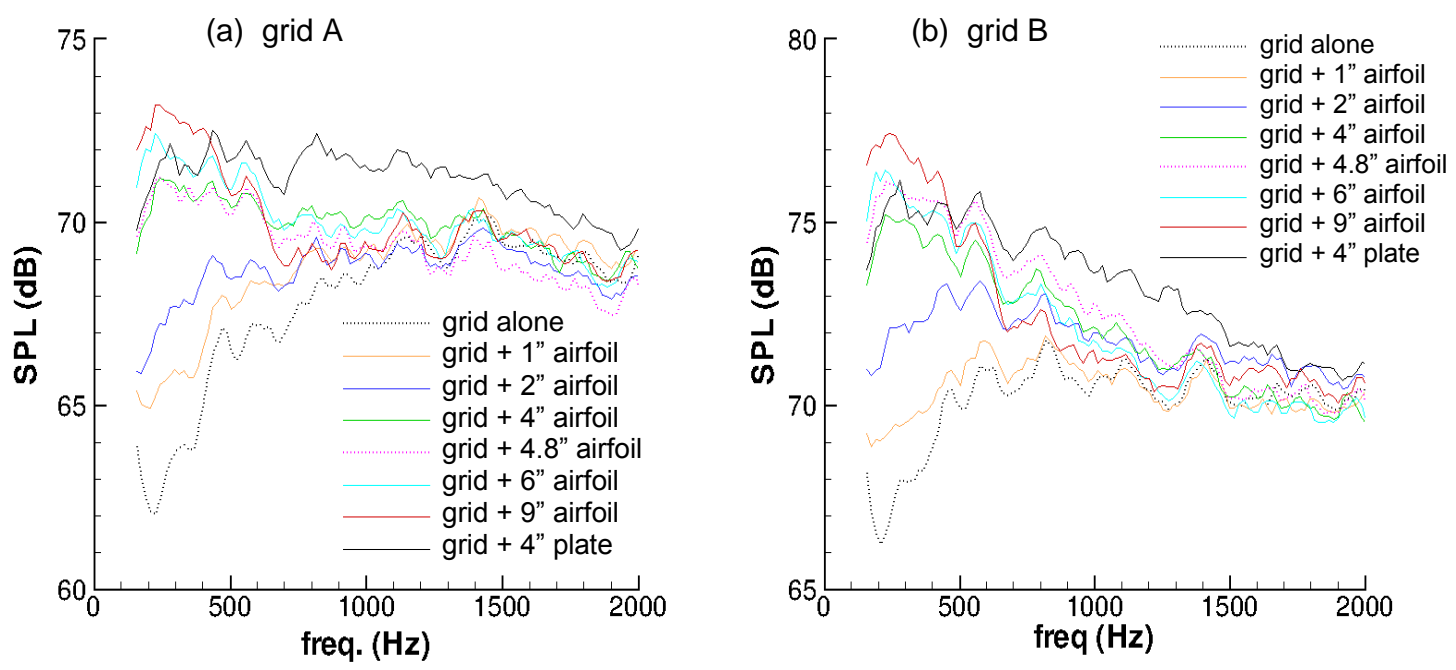

Figure 5. Raw signal from microphone M10 $(\Phi=\mathbf{- 9 0})$ for each turbulence grid. 

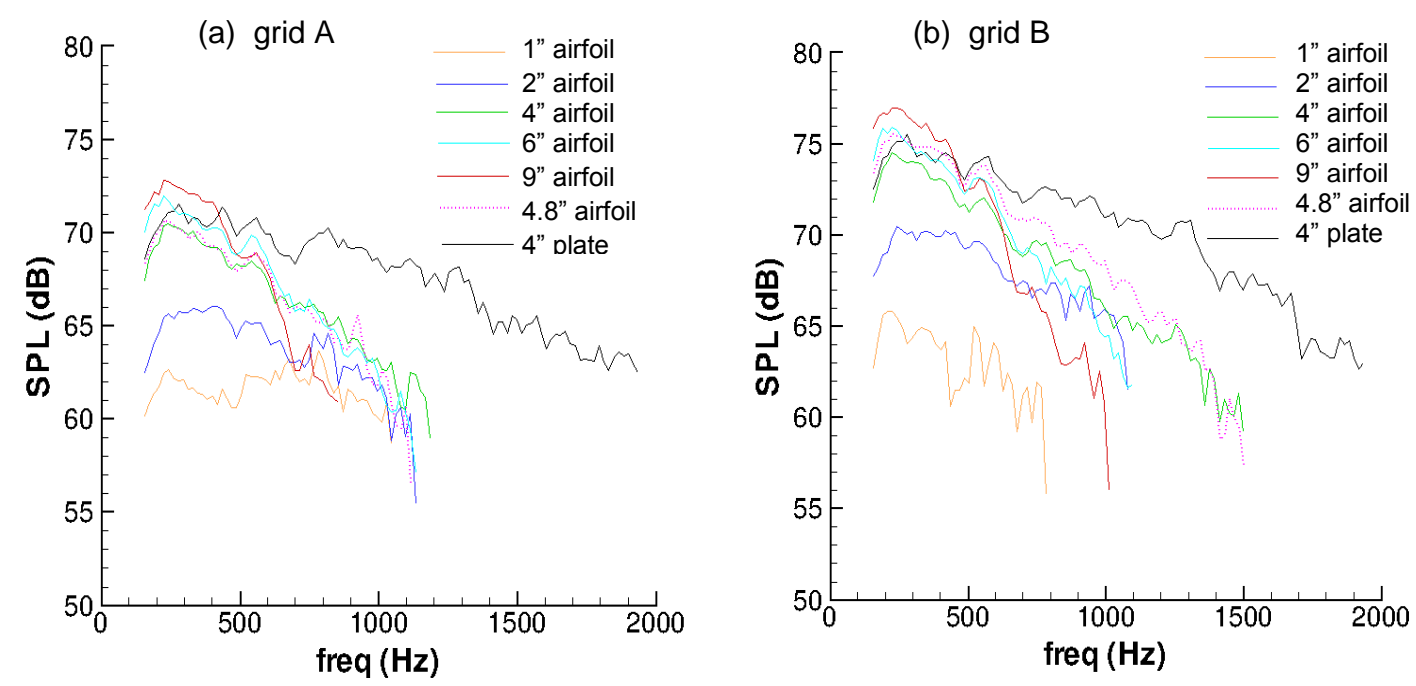

Figure 6. Auto-spectra from microphone M10 $(\Phi=\mathbf{- 9 0})$ after background noise subtraction.

\section{B. Laminar boundary layer vortex shedding noise}

Steps were taken to ensure that laminar boundary layer vortex shedding noise did not occur when the models were immersed in the grid generated turbulence. Laminar boundary layer vortex shedding noise is caused by Tollmien-Schlichting instability waves that can form if the boundary layer is laminar, and result in large contaminating quasi-tones (related to the vortex shedding rates at the trailing edge) in the noise spectra. An example of such tones is shown in Figure 7(a). This data was obtained from the un-tripped 4" chord airfoil immersed in a smooth flow (i.e. no turbulence grid) and positioned at a $5^{\circ}$ angle of attack.
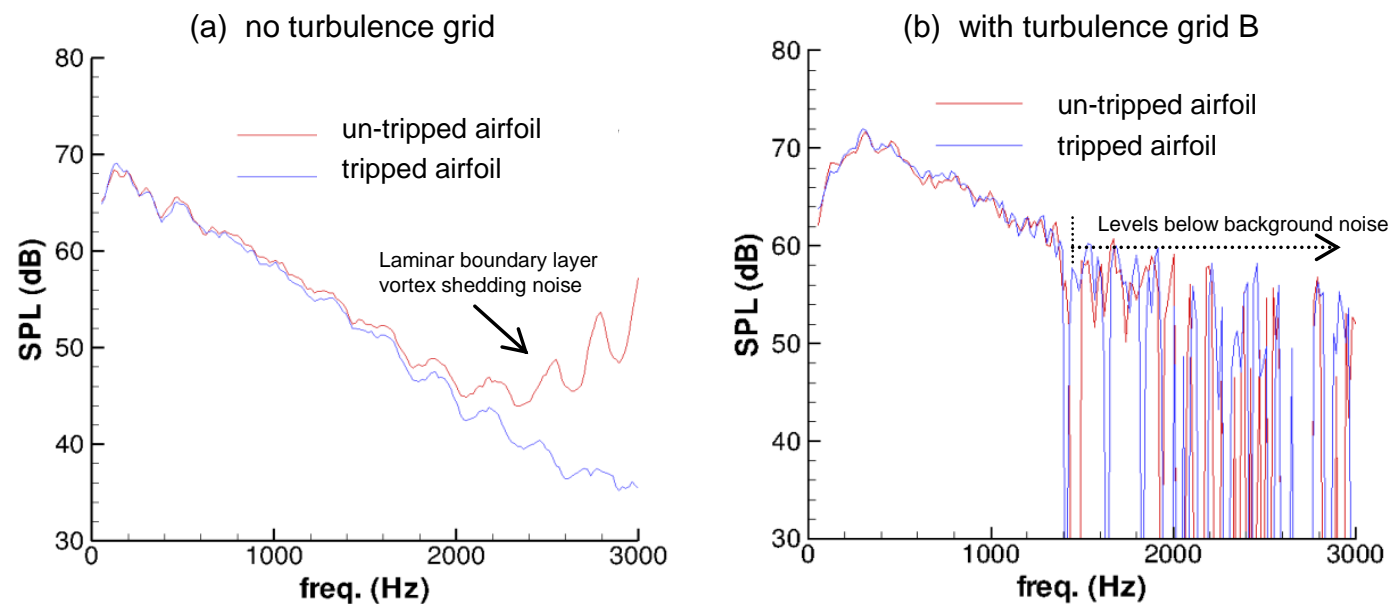

Figure 7. Microphone M10 auto-spectra for 4" NACA0015 airfoil at $\alpha=5^{\circ}$ and flow Mach number 0.127 .

The laminar boundary layer vortex shedding noise is seen to affect the spectra mostly above $1500 \mathrm{~Hz}$. Occurrences of laminar boundary layer vortex shedding noise could also be observed at various angles of attack for the other airfoils when tested un-tripped and in the absence of incident turbulence. In all instances, the laminar boundary layer vortex shedding noise was found to affect the spectra mostly above 
$1500 \mathrm{~Hz}$, i.e., somewhat outside of the frequency range where the turbulence interaction noise was measureable. Nevertheless, it was verified that for all test models, the laminar boundary layer vortex shedding noise was eliminated when the models were tripped. It was also verified that the spectra obtained with the tripped and un-tripped test models in the presence of incident turbulence were nearly identical. This indicates that the incident turbulence triggered the transition of the boundary layer to a turbulent state, eliminating the possibility of laminar boundary layer vortex shedding noise generation. The effect of the trip on the noise spectra for the 4 " airfoil at $5^{\circ}$ angle of attack is shown in Figure 7 (a). The good agreement between the spectra obtained from the tripped and un-tripped models with incident turbulence is shown for the same test case in Figure 7 (b). The data acquired with the un-tripped models was used to conduct the remainder of the analysis.

\section{Junction noise}

Microphone array measurements were performed for a small subset of test configurations to examine the possible contribution from extraneous noise at the junction of the models with the test section walls. The results obtained are only qualitative since the measurements were performed over a frequency range with corresponding beamwidth that is outside of the MADA design range. Thus, at $5 \mathrm{kHz}$, the array beamwidth is $1 \mathrm{ft}$, but at $1 \mathrm{kHz}$ it increases to approximately $5 \mathrm{ft}$. DAMAS processing [20] was used to obtain noise source localization maps. The maps were obtained by first generating DAMAS maps at each narrowband frequency between 710 and $1800 \mathrm{~Hz}$. The narrow band results were then summed on a pressure-squared basis to obtain the result shown. The map obtained for the 4 " airfoil at $0^{\circ}$ angle of attack and a flow Mach number of 0.127 is shown in Figure 8. It indicates the absence of dominant noise sources at the junctions and instead reveals the expected distributed nature of the noise sources which are seen located along the airfoil leading edge region.

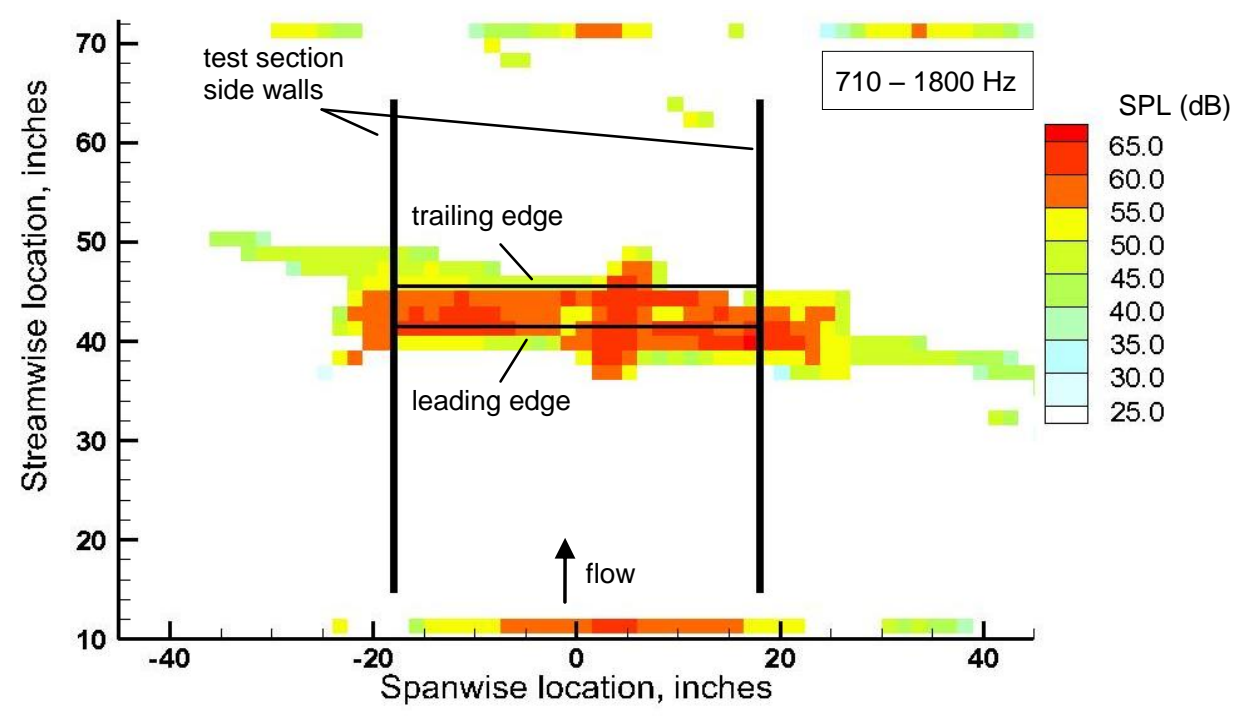

Figure 8. DAMAS noise source distribution for the 4" airfoil at $0^{\circ}$ angle of attack and a flow Mach number of 0.127. Incident turbulence generated by grid $B$. Frequency range is 710 to $1800 \mathrm{~Hz}$.

\section{Trailing edge noise and Source Location of Incident Turbulence Interaction Noise}

The cross-spectral phase of the acoustic signals obtained from the microphones positioned at $\Phi= \pm 90^{\circ}$ (microphones M3 and M10, referring to Figure 4) was examined for all of the airfoil configurations to determine the provenance of the measured noise and more specifically to verify that trailing edge (TE) noise sources from the turbulent boundary layer did not affect the noise spectra. The cross-spectral phase obtained for the 2", 4"and 9" NACA0015 airfoils and the 4.8" cambered airfoil, at $0^{\circ}$ and $20^{\circ}$ angles of 
attack, with turbulence grid B, and a flow Mach number of 0.127 are displayed in Figure 9. Note that effective angles of attack $\alpha^{*}$ (calculated in the manner described in Ref. 17) are indicated in parentheses.

It is seen that when the airfoils are at $0^{\circ}$ angle of attack, the cross-spectral phase remains around $180^{\circ}$ over the full measureable frequency range. This is consistent with the dipole nature of the turbulence interaction (and trailing edge) noise. At $\alpha=20^{\circ}$, the signals between microphones M3 and M10 remains strongly coherent and the shift in phase is found to be consistent with a source positioned downstream of the airfoil leading edge between $25 \%$ and $30 \%$ chord which is near the center of lift for the NACA0015 airfoils. This was also observed at intermediate angles of attack. This phase behavior is consistent with the whole airfoil radiating as a dipole.

The cross-spectral phase, $\varphi_{\mathrm{x}}$, that would be obtained for a dipole source centered on the airfoil at $\mathrm{x} \%$ chord can be expressed as

$$
\varphi_{\mathrm{x}}(\mathrm{f})= \pm \pi+\mathrm{k}\left(\mathrm{R}_{\mathrm{x}, \mathrm{M} 3}-\mathrm{R}_{\mathrm{x}, \mathrm{M} 10}\right)
$$

where $\mathrm{f}$ is the frequency, $\mathrm{k}$ is the wavenumber and $\mathrm{R}_{\mathrm{x}, \mathrm{Mj}}$ is the distance from microphone $\mathrm{Mj}$ to the $\mathrm{x} \%$ chord location. Using Eq. (3), the cross-spectral phases that would be obtained for a dipole source centered at the airfoil LE, at $25 \%$ chord, or at the airfoil TE, when the airfoil is at $20^{\circ}$ angle of attack, are shown as lines in Figure 9 for reference. It clearly shows that TE sources have little influence on the noise spectra.

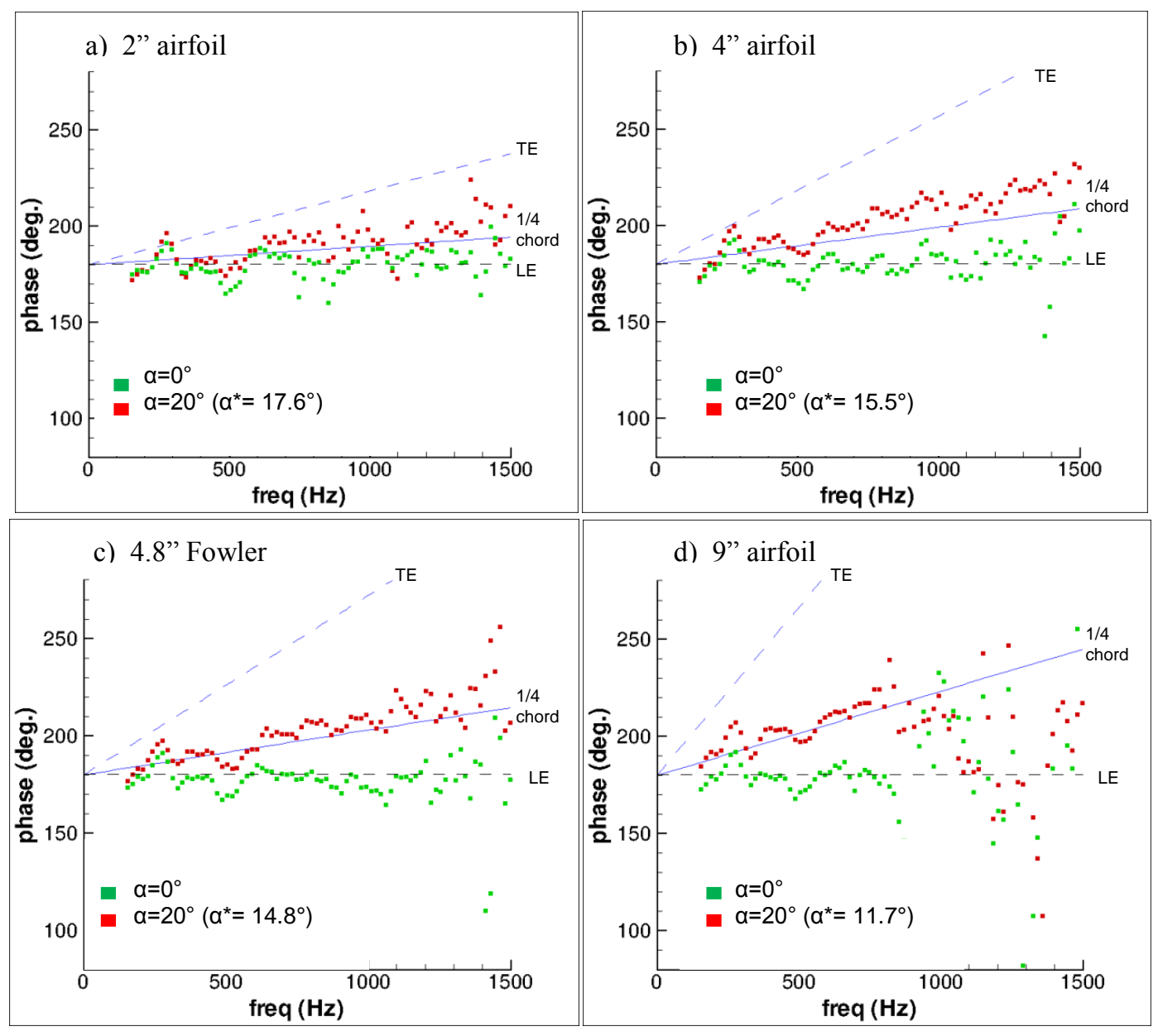

Figure 9. Cross-spectral phase between $\Phi= \pm 90^{\circ}$ microphones (i.e., M3 and M10). Incident turbulence generated by grid $B$, flow mach number is 0.127 . Keys: - cross-spectral phase at $\alpha=0^{\circ}$; $n$ cross-spectral phase at $\alpha=20^{\circ}$; source at $25 \%$ chord; source at Trailing Edge (TE); source at leading edge (LE). 


\section{E. Incident turbulence interaction noise spectra measurement}

Noise spectra obtained using the two approaches described in section 3 are compared. Results obtained for the 4 " airfoil at $0^{\circ}$ and $20^{\circ}$ angles of attack are shown in Figure 10. It is seen that the spectra obtained with the COP method (Eq. (1)) are within $1 \mathrm{~dB}$ of the spectra obtained directly from the microphone signals auto-spectra (Eq. (2)). This demonstrates the dipole characteristic of the noise generated by the incident turbulence, and that the cross-spectrum $\mathrm{G}_{\mathrm{M} 3, \mathrm{M} 10}$ appears to properly capture the dipole strength (i.e., the interaction noise). The agreement obtained between the two methods also indicates that, above background noise levels, the incident turbulence interaction noise dominates the noise field and can be directly measured from the microphone signals auto-spectra. In the rest of the analysis, the noise spectra presented were obtained from Eq. (2).
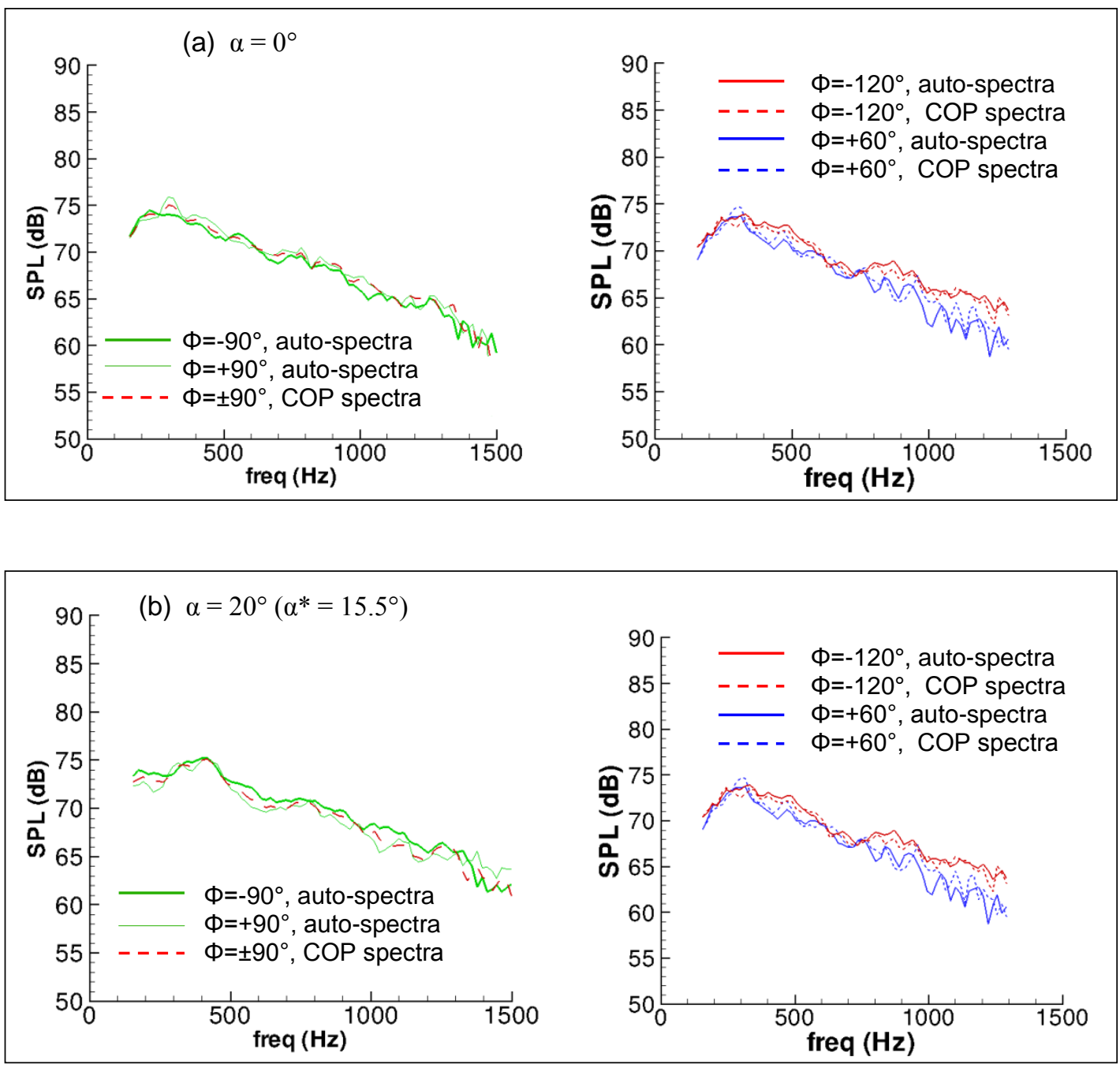

Figure 10. Comparison between spectra obtained directly from microphone auto-spectra and spectra obtained through the COP method. 4" NACA0015 airfoil, turbulence grid B and flow Mach number of 0.127.

Keys: - - - - obtained using Eq. (1); _ obtained using Eq. (2). 


\section{F. Effect of airfoil chord and leading edge radius on noise spectra}

The characteristics of the noise spectra obtained at $\Phi=90^{\circ}$ (microphone M10) for the different model tested are compared. The models were positioned at $0^{\circ}$ angle of attack, and the flow Mach number was 0.127. The one-third octave band spectra obtained with turbulence grids A and B are shown in Figures 11 and 12, respectively (spectra were also shown in Figure 6 in narrowband frequencies). It is seen from these figures that as the NACA0015 airfoil LE radius increases (proportionally to the airfoil chord), the spectral levels at high frequencies are more reduced. This is consistent with findings from previous studies and with the observation that as the airfoil LE radius increases to a larger size, the sectional lift fluctuation (near the airfoil LE) resulting from impingement by the smaller eddies (which are responsible for the higher frequency content of the spectra) is not as efficiently developed and the associated noise production is reduced. The noise spectrum obtained with the flat plate illustrates the limiting case of a "near zero" LE radius.

It is also seen from these figures that as the model chord increases, the spectral peak level increases. With the exception of the cambered airfoil, for each doubling of the chord, the spectral peak level increased by approximately 3 to $6 \mathrm{~dB}$ with the larger increase occurring for the thinner airfoils. This is consistent with Paterson and Amiet's analytical model for sound radiation from an airfoil in a turbulent flow and an observer in the airfoil mid-span plane (Eq. (15a) of Reference 2), which (in the low frequency limit) indicates a $6 \mathrm{~dB}$ increase in SPL per doubling of the chord. Paterson and Amiet's model, however, assumes that the airfoil is a flat plate of zero thickness, which may explain the reduced increase in spectral levels observed as the airfoils thickness increased. This observation is supported by the fact that the difference in peak levels seen between the 2" and 4" NACA0015 airfoils (of thicknesses 0.3 " and 0.6", respectively) is approximately 4 to $5 \mathrm{~dB}$, while that between the 2" NACA0015 and the 4" plate (of same maximum thickness) is closer to $6 \mathrm{~dB}$.

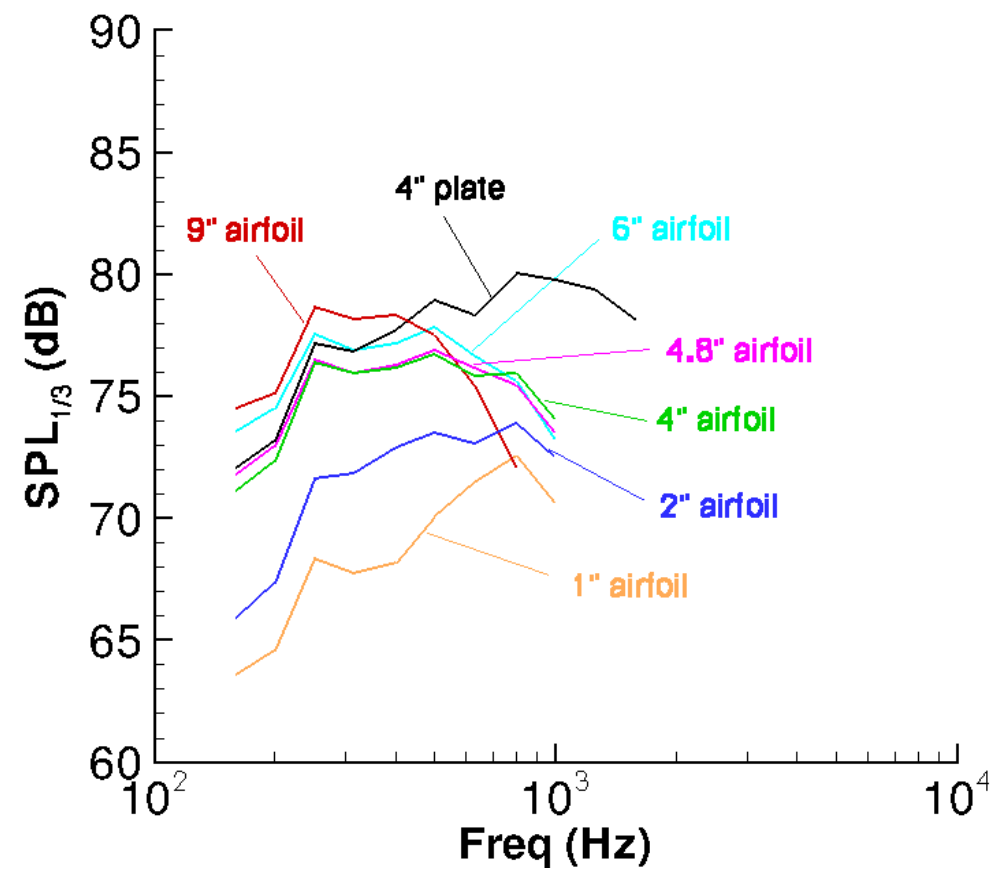

Figure 11. One-third octave band auto-spectra from microphone M10 (i.e., $\Phi=-90^{\circ}$ ). Turbulence grid A, Mach number 0.127, $\alpha=0^{\circ}$. 


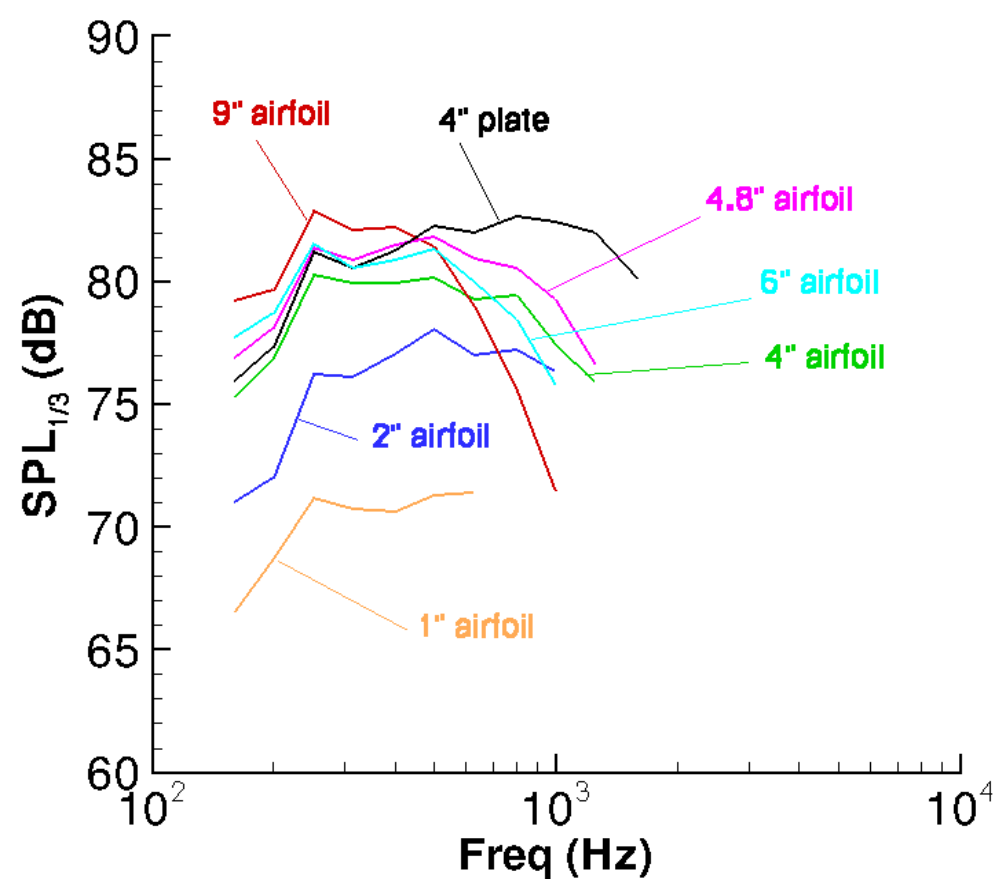

Figure 12. One-third octave band auto-spectra from microphone M10 (i.e., $\Phi=-90^{\circ}$ ). Turbulence grid B, Mach number $0.127, \alpha=0^{\circ}$.

Hence, for the symmetric airfoils and flat plate model tested, the spectral peak level appears to be mostly controlled by the airfoil's thickness and chord, while the degree of decrease in the spectral level at higher frequencies appears to be controlled by the model's LE sharpness. It is noted that when only taking into consideration LE radius, chord and thickness, the data obtained for the cambered airfoil did not follow the trends noted above as consistently. The same behavior was observed with the cambered airfoil positioned at $\alpha=-5^{\circ}\left(\alpha^{*}=-3.7^{\circ}\right)$, an angle of attack that is near the airfoil's "zero lift" configuration.

\section{G. Effect of turbulence intensity and integral scales}

Increase in turbulence integral scale and intensity was found to lead to a uniform increase of the LE noise spectral levels without significantly affecting other spectral characteristics (such as peak frequency or rate of decrease in levels with frequency). Noise spectra obtained from the different model configurations with turbulence grids A and B are compared in Figure 13. It is seen that switching from turbulence grid A to turbulence grid B leads to an increase of 3 to $4 \mathrm{~dB}$ in spectral level for the NACA0015 airfoils and the flat plate. This shift in level is again consistent with Amiet's model for the prediction of SPL from an airfoil in a turbulence stream (Eq. 28 of Reference 1). In his model, for a given airfoil and flow Mach number, SPL scales with $10 \log \left(\mathrm{LI}^{2}\right)$. Referring to Table 1, this corresponds to an increase of $3.2 \mathrm{~dB}$ which is very consistent with the experimental data shown in Figure 13. The change in turbulence scale and intensity was more significant for the cambered airfoil, with an increase of at least $5 \mathrm{~dB}$. Further investigation is needed to fully explain this difference. 
(a) 2" NACA0015
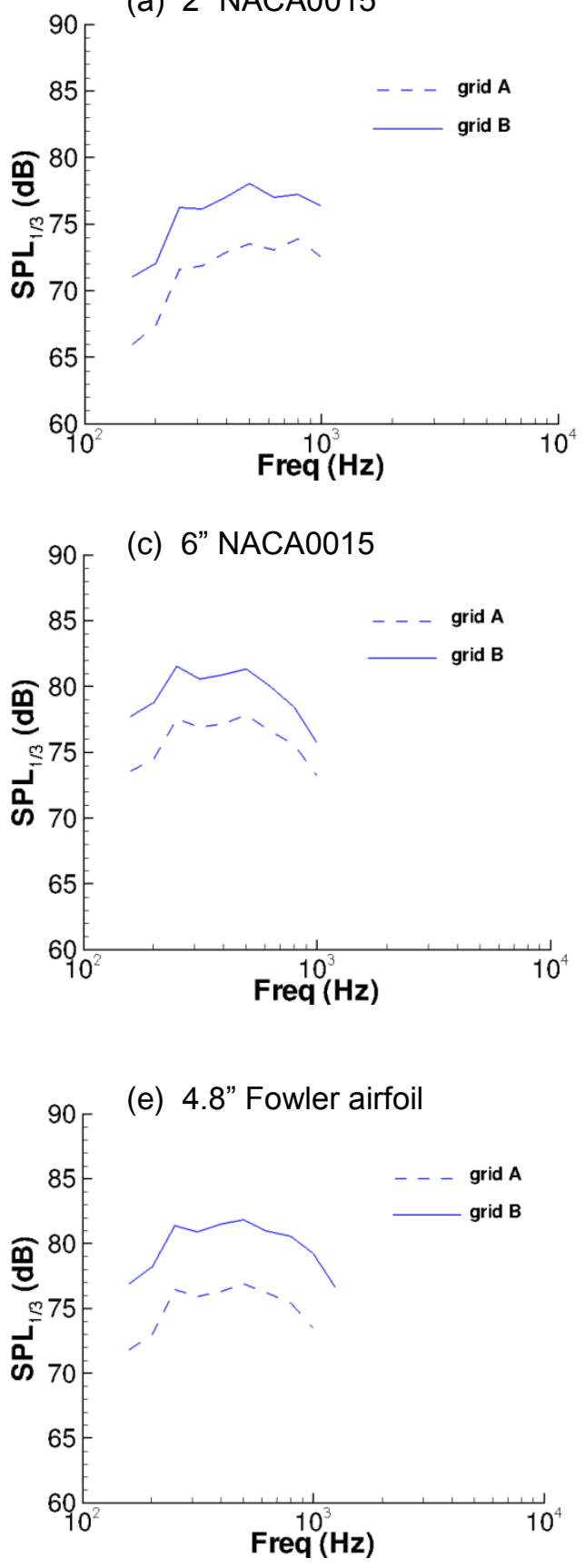
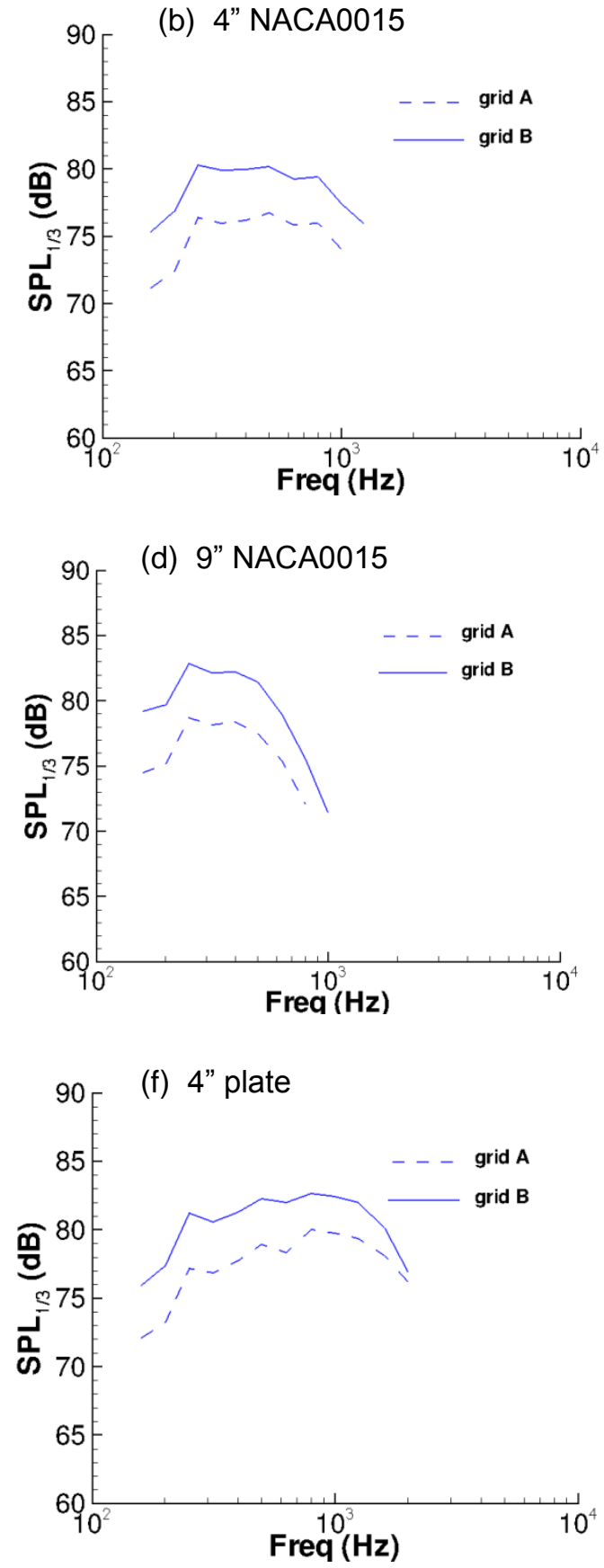

Figure 13. Change in levels due to change in turbulence scale and intensity. 


\section{H. Effect of flow speed}

The velocity dependence of the incident turbulence interaction noise was examined for all the airfoil models tested, and an optimum $6^{\text {th }}$ power level dependence on velocity was found. This result is in good agreement with Oelermans and Migliore [Ref. 9] experimental observations, and with theoretical predictions [Ref. 2] at low frequencies, where the acoustic wavelength is much larger than the airfoil chord (as is the case in this study). The spectra obtained for the 4" NACA0015 airfoil at $\Phi=90^{\circ}$ for $\alpha=0^{\circ}$ and $10^{\circ}$ are presented in Figures 14 and 15, respectively. The incident turbulence was generated by grid B. The spectra are normalized with the $6^{\text {th }}$ power of Mach number and in a Strouhal fashion where only Mach number is varied.
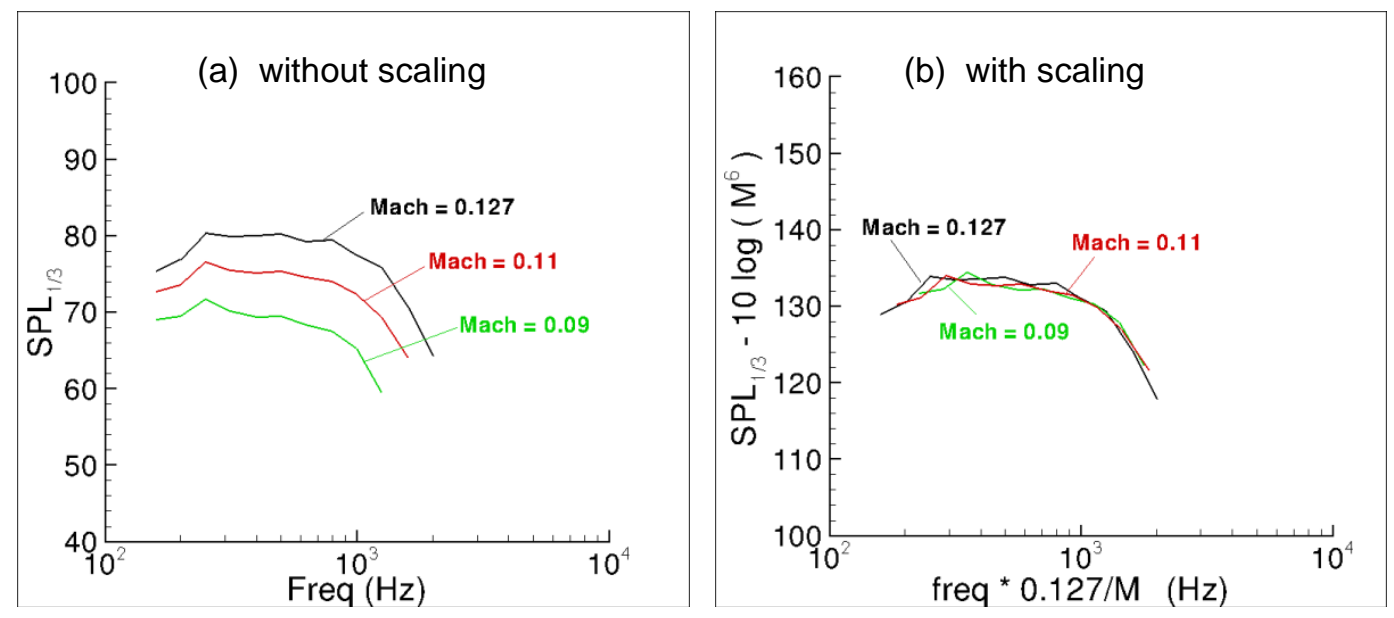

Figure 14. Effect of flow speed. 4 " airfoil, grid $B, \alpha=0^{\circ}$.
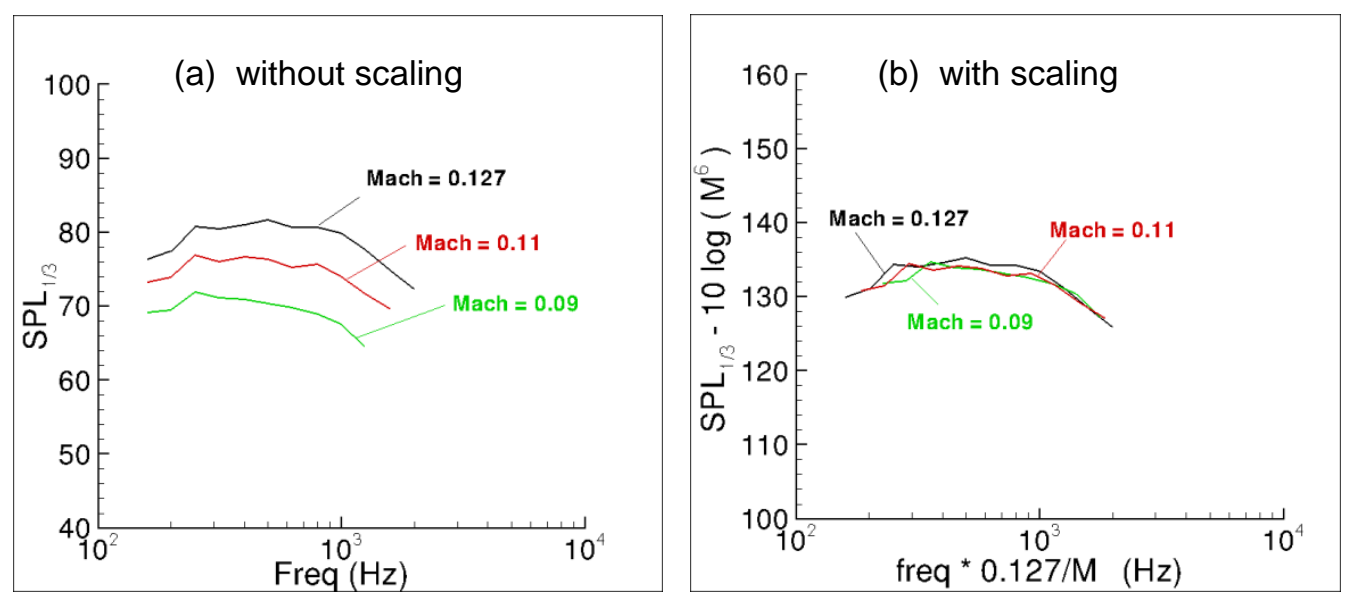

Figure 15. Effect of flow speed. 4 " airfoil, grid $B, \alpha=10^{\circ}$. 


\section{Directivity and effect of angle of attack}

The effect of angle of attack on the noise directivity was examined. The spectra for the 1", 2", 4" and 9" NACA0015 airfoils, and 4.8" cambered airfoil at Mach 0.127, obtained from the different microphone locations on both the suction and pressure sides of the airfoils, are presented in Figures 16,17, 18, 19 and 20 , respectively. The turbulence was generated with grid B. Consistent results were obtained for the NACA0015 airfoil suction side and pressure side directivity data whether obtained with the airfoils positioned at negative or positive $\alpha$. Theoretical compact dipole and baffled dipole directivity patterns are shown in some of the figures for reference. For the compact dipole, the theoretical directivity peak levels are normalized to the measured levels at the corresponding emission angle while the baffle dipole directivity is positioned to pass through the peak of the compact dipole curve.

The noise directivity in a frequency range about the spectral peak and in a higher frequency range were examined separately and are presented in separate plots. The sound pressure levels shown were obtained by summing the narrowband spectral levels between $140 \mathrm{~Hz}$ and $600 \mathrm{~Hz}$ for the spectral peak frequency range, and between $600 \mathrm{~Hz}$ and $1200 \mathrm{~Hz}(1000 \mathrm{~Hz}$ for the 9" airfoil) for the higher frequency range. The summations were performed on a pressure-squared basis. The upper limits of the higher frequency range were chosen such that the noise levels were above background noise levels at all the microphones. For the 1 " and 2" airfoils, only results obtained in the frequency range of the spectral peak are shown because background noise affected some of the data at higher frequencies.

Spectral levels and emission angles $\Phi_{\mathrm{e}}$ were corrected to account for shear-layer effects in the manner shown in Ref. 20. For simplicity, to calculate shear layer corrections, a source location at the airfoil leading edge and a straight shear-layer (no curvature) were assumed. The latter assumption added uncertainty to the levels given for the 9" and 6"airfoils at large angles of attack, where the shear-layer would have had the most curvature.

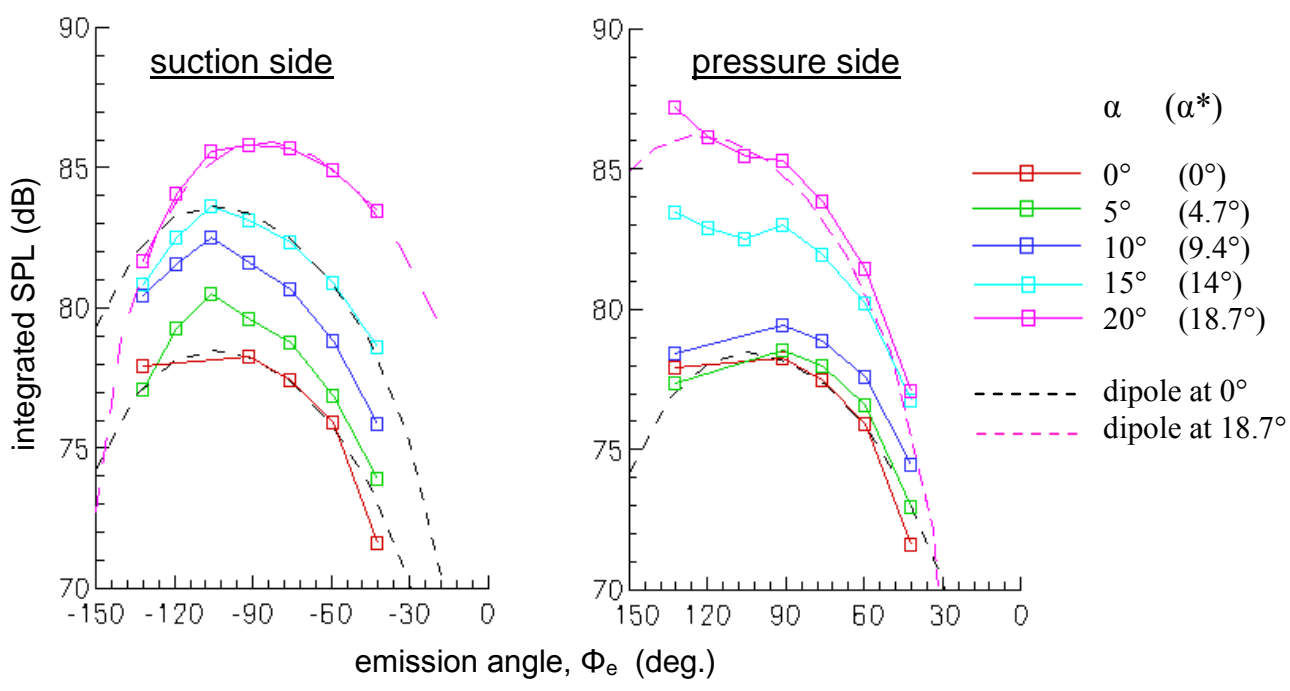

Figure 16. 1" airfoil directivity; Mach=0.127, grid B. Noise levels shown for the spectral peak frequency range. $\Phi_{\mathrm{e}}$ measured from downstream direction. 
It is seen from Figure 16 that for the 1" airfoil, an increase in angle of attack leads to a significant increase in noise. Namely up to $4 \mathrm{~dB}$ at an effective angle of attack, $\alpha^{*}$, of $9.4^{\circ}, 5 \mathrm{~dB}$ for $\alpha^{*}=14.0^{\circ}$ and up to $8 \mathrm{~dB}$ for $\alpha^{*}=18.7^{\circ}$. Angle of attack effects also appear to be more significant on the model suction side than pressure side at smaller $\alpha^{*}$ values. In comparison, for the 2" airfoil, a significant noise increase (3 to 4 $\mathrm{dB}$ ) with angle of attack was only observed for $\alpha^{*}=17.6^{\circ}$. Only 1 to $2 \mathrm{~dB}$ level changes for the $2^{\prime \prime}$ airfoil were observed for smaller $\alpha^{*}$ angles. Referring to Table 2, it is noted that for the 1" airfoil, the turbulence integral scale is larger than the airfoil chord $(\mathrm{L} / \mathrm{C}=1.46)$ while for the 2 " airfoil, the turbulence integral scale is smaller than the airfoil chord $(\mathrm{L} / \mathrm{C}=0.73)$.

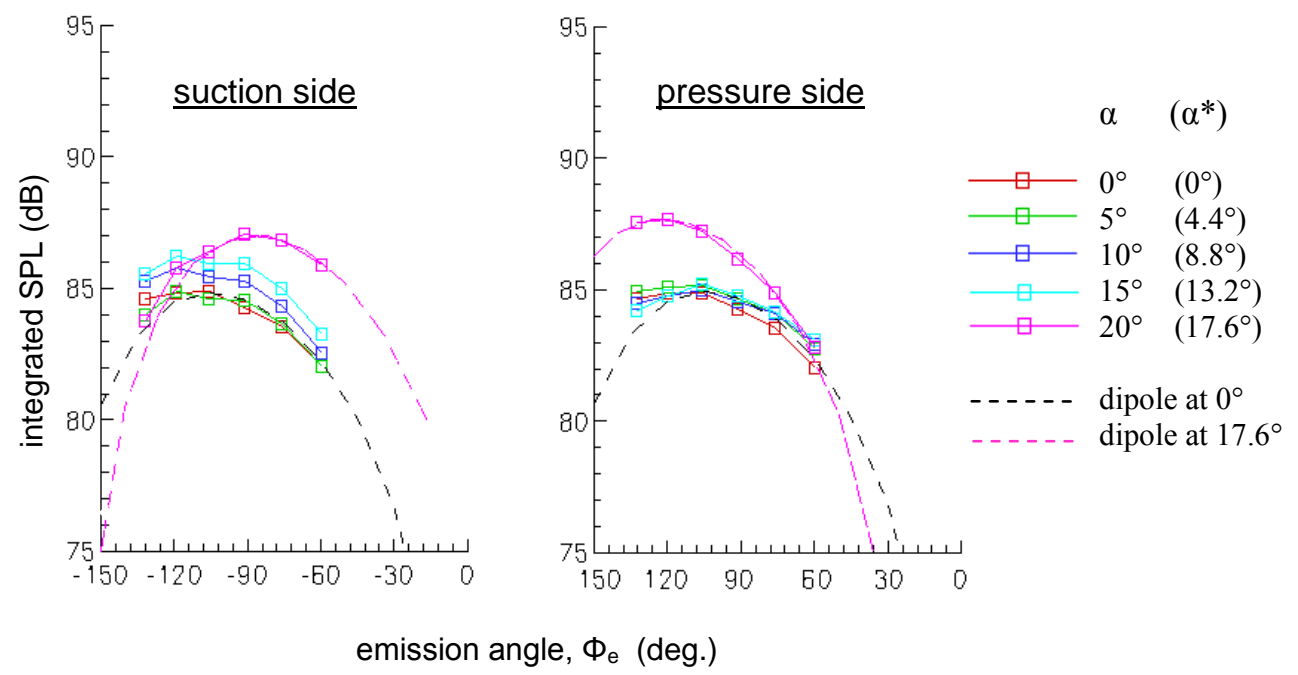

Figure 17. 2" NACA0015 directivity; Mach=0.127, grid B. Noise levels shown for the spectral peak frequency range. $\Phi_{\mathrm{e}}$ measured from downstream direction.

It is seen from Figures 18, 19 and 20 that at corresponding $\alpha^{*}$, the level changes with angle of attack for the larger airfoils (reduced L/C) are much less than that seen for the 1" airfoil. Only a 2 to $3 \mathrm{~dB}$ increase is observed for the larger airfoils over the higher frequency range of the noise spectra, while a 1 to $2 \mathrm{~dB}$ increase is seen over the frequency range of the spectral peak. The angle of attack effect appears to be also more pronounced on the models suction side than on the pressure side.

With the exception of the $\alpha=20^{\circ}$ case for the 1 " and 2 " airfoils, the directivity patterns in the frequency range of the spectral peak were observed to mostly follow that of a compact dipole at $0^{\circ}$ angle of attack even as the airfoil angle of attack changed. This indicates that the directivity tends to remain symmetric with respect to the direction of the mean flow. In contrast, the directivity patterns for the 1" and the 2" airfoils at the largest angle of attack tested were observed to follow that of a compact dipole positioned at the same angle of attack (i.e., the directivity is symmetric to the airfoil chord). It is believed that this change in directivity is due to the 1 " and 2" airfoils being stalled at $\alpha=20^{\circ}$. 
a) spectral peak frequency range

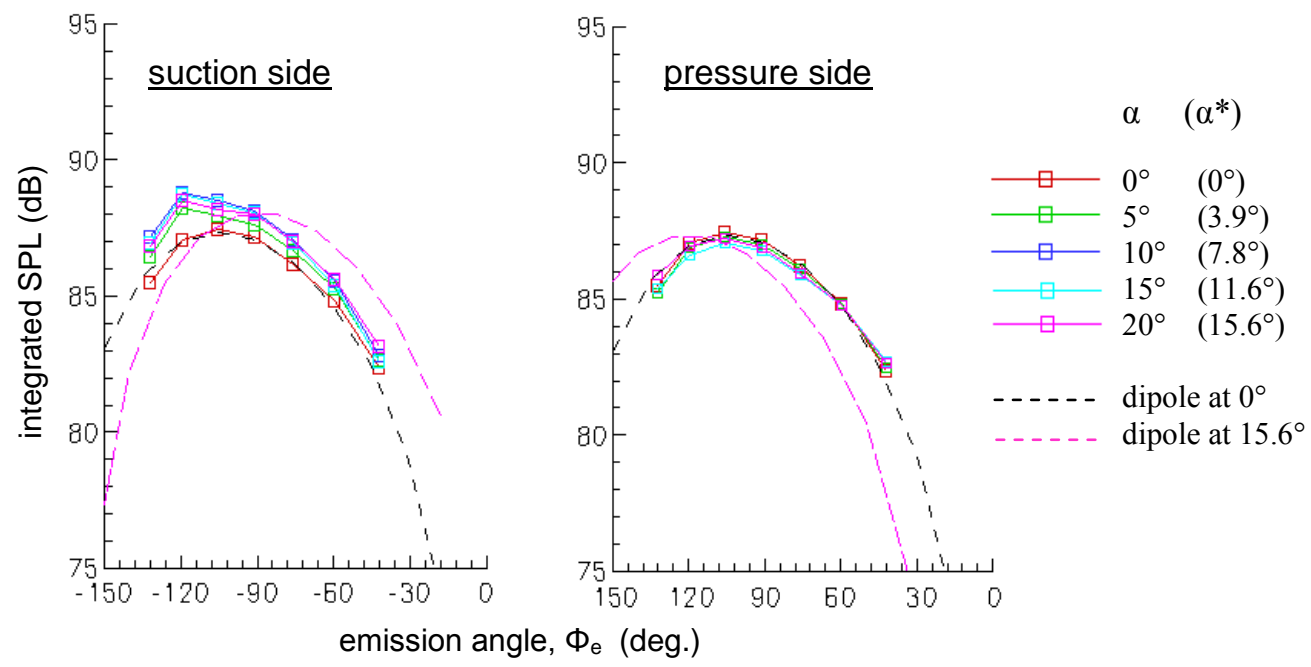

\section{b) high frequency range}
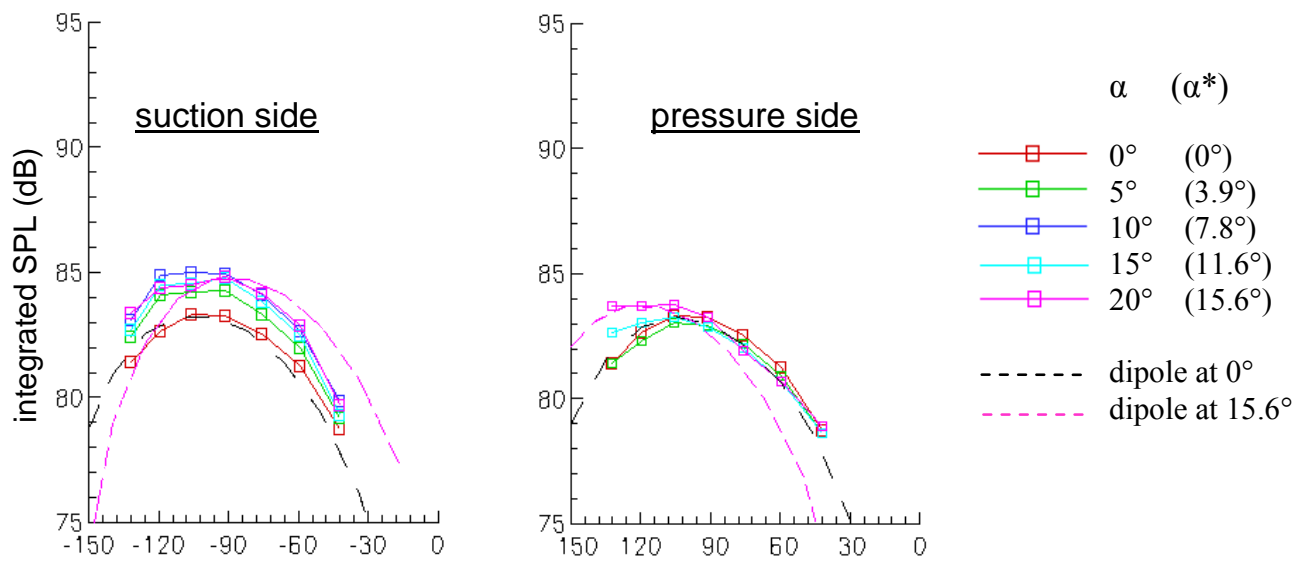

emission angle, $\Phi_{\mathrm{e}}$ (deg.)

Figure 18. 4" NACA0015 directivity; Mach=0.127, grid B. Noise levels shown for a) the spectral peak frequency range, b) high frequency range.

Shear-layer corrected emission angle $\Phi_{\mathrm{e}}$ measured from downstream direction.

Finally, for the largest airfoils tested, namely the 6" and 9" NACA0015 airfoils, the directivity pattern in the higher frequency range of the spectra did not follow that of a compact dipole as well as the smaller airfoils did (this is seen in Figure 19 (b) for the 9" airfoil). Instead, the directivity pattern is seen to tend toward the pattern associated with a baffled dipole, as suggested by the spectral peak levels remaining elevated further downstream. This behavior was also observed by Paterson and Amiet [Ref. 2] and is consistent with the assumption that as the wavelength of the turbulent gusts become small with respect to the airfoil chord (high frequencies), the airfoil chordwise pressure is no longer in phase and the loading 
tends to concentrate near the leading edge. Although a full transition to a baffle directivity pattern would have likely been observed for larger airfoil chords, in the present study, all of the airfoils tested could be modeled as compact dipoles. It was however previously noted that the angle of attack effect appears to be more pronounced on the model's suction side than on the pressure side, which would not be expected for a simple dipole. The higher levels seen on the suction side may be related to a near separation area where the interaction of the turbulence eddies with the airfoil surface is increased, leading to increased noise. This merits further study.

It may be drawn from the above analysis that, in the absence of stall, the directivity of incident turbulence interaction noise remains symmetric with respect to the direction of the mean flow, and that angle of attack changes have a significant effect on noise levels when the incident turbulence integral scale is larger than the airfoil chord. However, the latter observation would also require further investigation as only one test model, in the present study, fit that criterium.

\section{a) spectral peak frequency range}

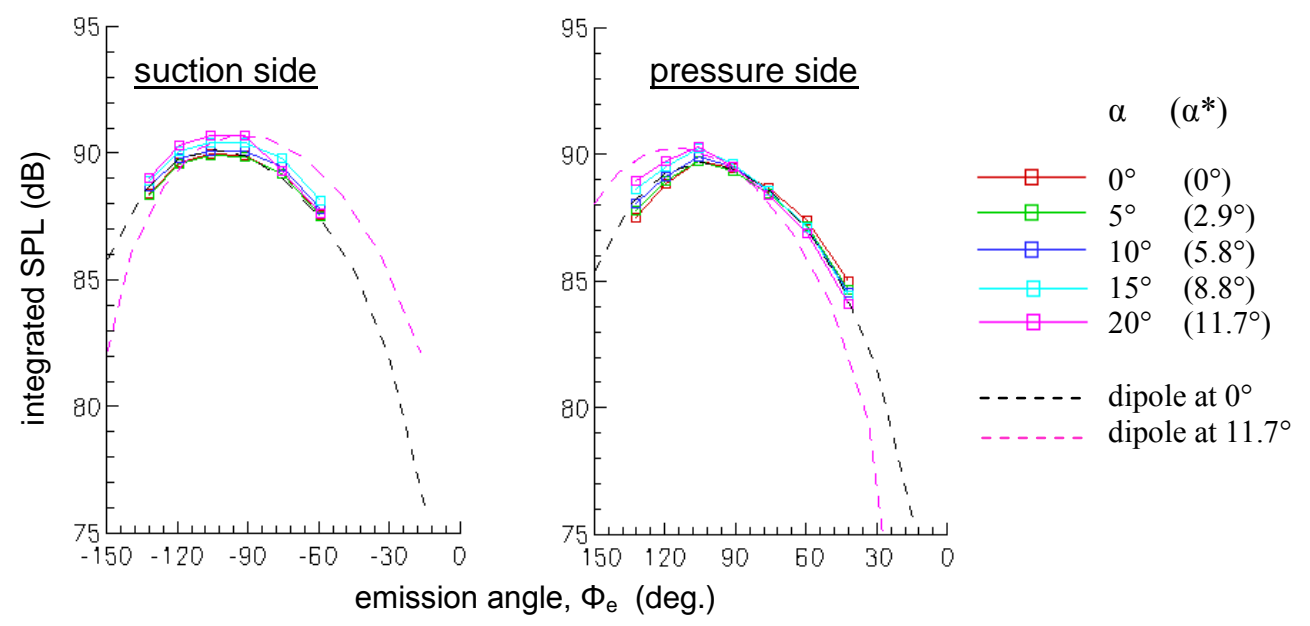

b) high frequency range

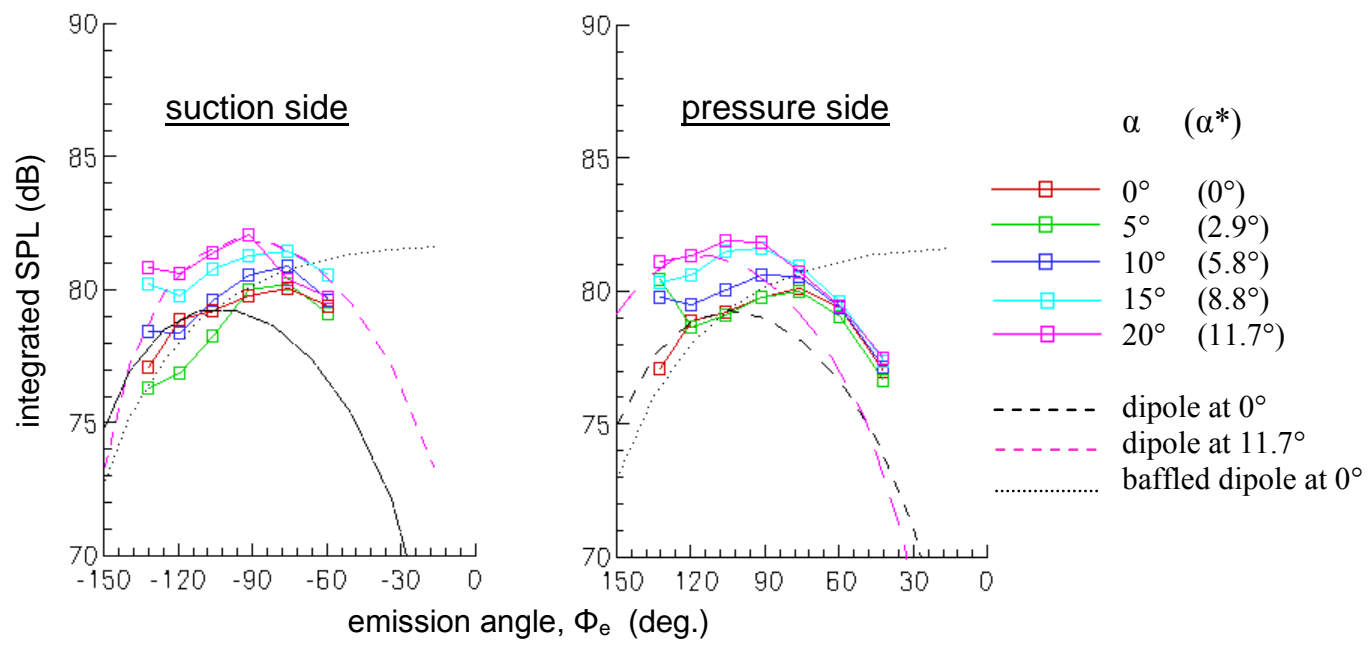

Figure 19. 9" NACA0015 directivity; Mach=0.127, grid B. Noise levels shown for a) the spectral peak frequency range, $b$ ) high frequency range. Shear-layer corrected emission angle $\Phi_{\mathrm{e}}$ measured from downstream direction. 


\section{a) spectral peak frequency range}

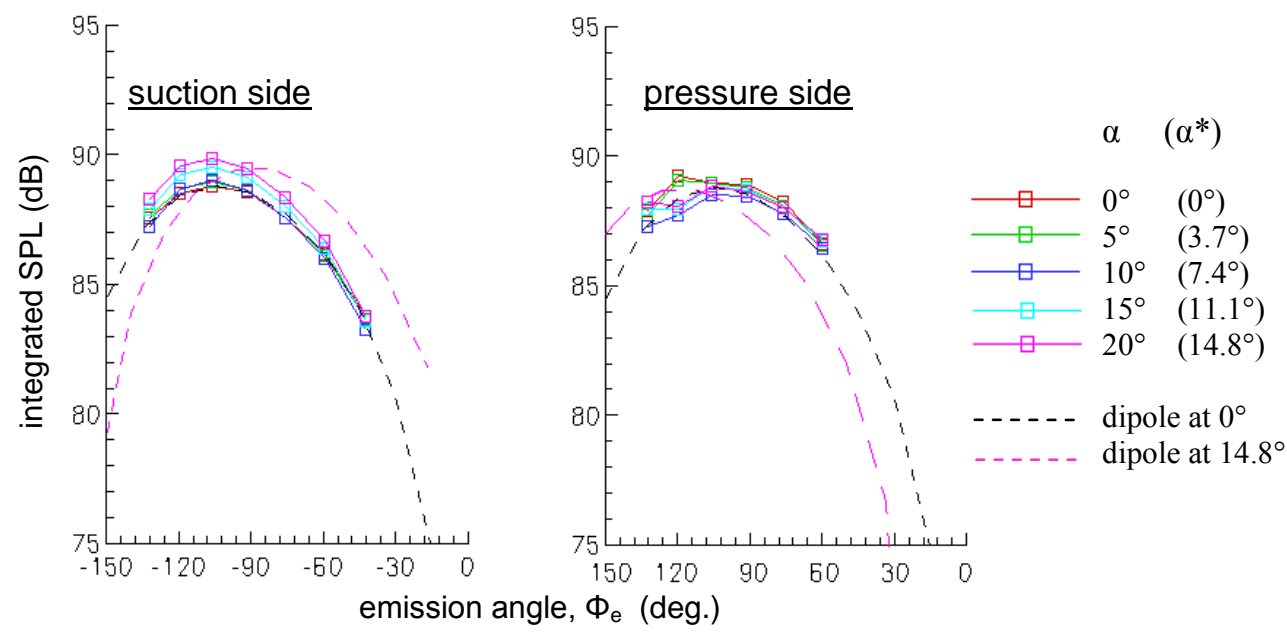

b) high frequency range

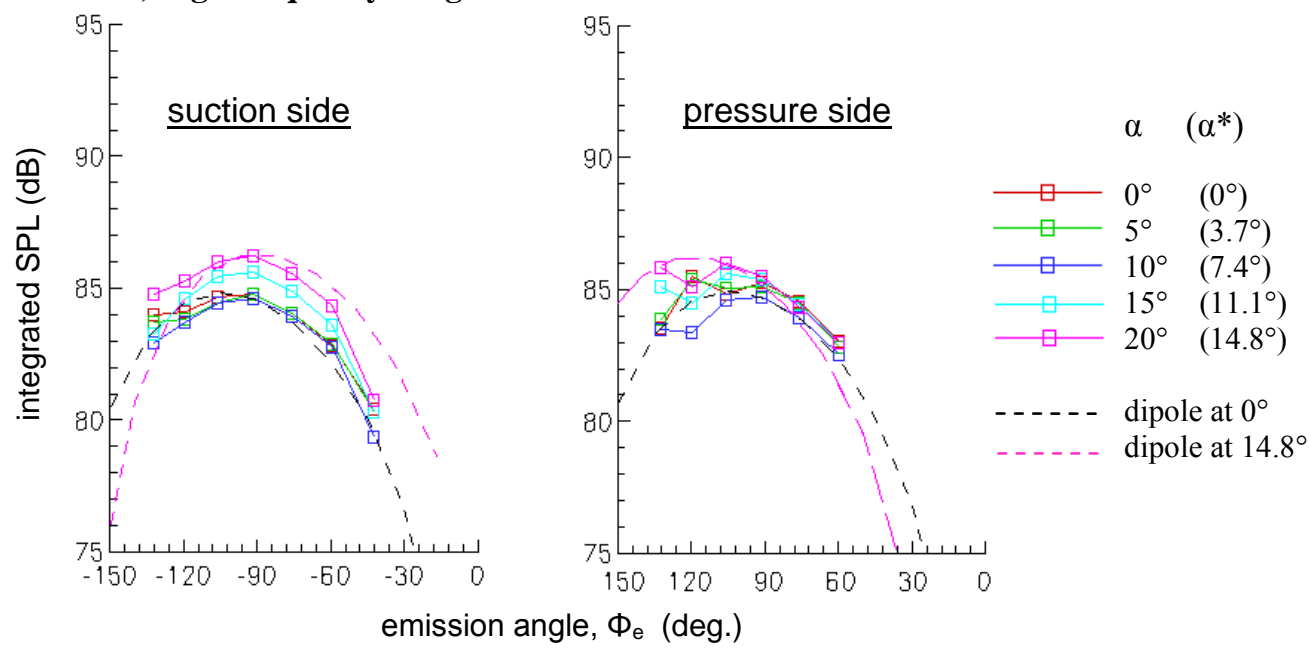

Figure 20. 4.8" airfoil directivity; Mach=0.127, grid B. Noise levels shown for a) the spectral peak frequency range, b) high frequency range.

Shear-layer corrected emission angle $\Phi_{\mathrm{e}}$ measured from downstream direction.

\section{Summary}

Detailed directivity measurements of noise resulting from the interaction with incident turbulence were obtained on a series of NACA0015 airfoils, a flat plate and a cambered airfoil section. The incident turbulence characteristics are described. Two approaches for performing the noise measurements were evaluated. The first one (COP method) is based on the cross-spectral analysis of output signals from microphones placed around the test models. In the second approach, noise spectra are obtained directly from the auto-spectra of output signals from individual microphones. The good agreement obtained 
between the two methods demonstrates the dipole characteristic of the noise generated by the incident turbulence.

The effects that turbulence intensity and integral length scales, airfoil geometry, velocity and angle of attack have on the resulting noise are examined. It is seen that noise levels at frequencies higher than the spectral peak decrease more as the airfoil LE sharpness decrease, and that the spectral peak levels (at $0^{\circ}$ angle of attack) appear to be mostly controlled by the airfoil's thickness and chord. Increase in turbulence longitudinal integral scale (L) and intensity (I) are found to lead to an increase of the noise spectral levels without significantly affecting other spectral characteristics. In agreement with theoretical predictions, a $6^{\text {th }}$ power level dependence on velocity and $2^{\text {nd }}$ power level dependence on airfoil chord are found. $\mathrm{An}^{\mathrm{LI}^{2}}$ dependence is also verified. Angle of attack is observed to have a significant effect on the noise levels for the smallest airfoil tested (approximately a $2 \mathrm{~dB}$ noise increase per 4 to 5 degrees angle of attack increase) and little effect for the larger airfoils (approximately $2 \mathrm{~dB}$ increase for a 10 to 15 degrees angle of attack increase). Angle of attack effects appear to have an integral scale to chord ratio (L/C) dependence, with large effects on noise for $\mathrm{L} / \mathrm{C} \geq 1$ and decreasing effects as $\mathrm{L} / \mathrm{C}$ becomes smaller than 1 . For all $\mathrm{L} / \mathrm{C}$ values, the directivity pattern of the noise resulting from the incident turbulence is seen to remain symmetric with respect to the direction of the mean flow until stall, at which point the directivity becomes symmetric with respect to the airfoil chord. Finally, it is observed that sensitivity to angle of attack is more pronounced on the model suction side than on the model pressure side (this observation merits further investigation). For the largest airfoils tested (L/C < 0.24), angle of attack effects are mostly seen at high frequencies.

\section{Acknowledgments}

The authors wish to thank Gerald E. Plassman for processing of the DAMAS noise maps and Dennis H. Kuchta and Jaye A. Moen for invaluable support in the experiment. The authors also gratefully acknowledge the support received from the Subsonic Fixed Wing and Subsonic Rotary Wing projects.

\section{References}

1. Amiet, R. K. "Acoustic radiation from an Airfoil in a Turbulent Stream", Journal of Sound and vibration (1975), Vol. 41(4).

2. Paterson, R. W., Amiet, R. K. "Noise and Surface response of an Airfoil due to Incident Turbulence", AIAA paper 76-571.

3. Casper, J., Farassat, F., Mish, P. and Devenport, W. "Broadband Noise Predictions for an Airfoil in a Turbulent Stream", AIAA paper 2003-366.

4. Moreau, S. and Roger, M. "Competing Broadband Noise Mechanisms in Low Speed Axial Fans", AIAA paper 2004-3039.

5. Glegg, S., Devenport, W. and Staubs, J. “Leading Edge Noise”, AIAA paper 2006-2424.

6. Glegg, S. Devenport, W. and Staubs, J. "Sound radiation from Three Dimensional Airfoils in a Turbulent Flow", AIAA paper 2008-52.

7. Mish, P., Wang, H., Bereketab and Devenport, W. " Measurement of the Space-Time Correlation of Surface Pressure Fluctuations on an Airfoil Immersed in Turbulence” AIAA paper 2000-2604.

8. Mish, P., Devenport, W. and Borgoltz, A. "Evaluation and Further Analysis of Unsteady Pressure Response Measurements on an Airfoil at Angle of Attack", AIAA paper 2002-2457.

9. Oerlemans, S. and Migliore, P. "Aeroacoustic Wind Tunnel Tests of Wind Turbine Airfoils", AIAA paper 2004-3042.

10. Migliore, P. and Oerlemans, S. "Wind Tunnel Aeroacoustic Tests of Six Airfoils for Use on Wind Turbine", AIAA paper 2004-1186. 
11. Moreau, S., Roger, M. and Jurdic, V. "Effect of Angle of Attack an Airfoil Shape on TurbulenceInteraction Noise", AIAA paper 2005-2973.

12. Staubs, J., Devenport, W. and Glegg, S. "Sound radiation from a series of Airfoils Immersed in GridGenerated Turbulence", AIAA paper 2008-3018.

13. Brooks, T. F. and Burley, C. L. "Blade Wake Interaction Noise for a Main Rotor", Journal of the American helicopter Society, January 2004.

14. Roach, P. E. "The generation of Nearly Isotropic Turbulence by means of Grids" Journal of Heat and Fluid Flow, Vol. 8(2), June 1987.

15. Brooks, T. F. and Hodgson, T. H. "Trailing Edge Noise Prediction from Measured Surface Pressures", Journal of Sound and Vibration (1981), Vol. 78(1).

16. Bendat, J. and Piersol, A., "Engineering Applications of Correlation and Spectral Analysis", John Wiley \& Sons, 1980.

17. Brooks, T. F., Pope, D. S., and Marcolini, M. A., "Airfoil Self-Noise and Prediction”, NASA Reference Publication 1218, July 1989.

18. Hinze, J. O., “Turbulence”, McGraw Hill, New York, 1975.

19. Olsen, W. A., "Noise Generated by Impingement of Turbulent Flow on airfoils of Varied Chord, Cylinders, and Other Flow Obstruction", AIAA Paper 76-504.

20. Brooks, T. F. and Humphreys, W. M., "A Deconvolution Approach for the Mapping of Acoustic Sources (DAMAS) Determined from Phased Microphone Arrays", Journal of Sound and Vibration (2006), p. 856-879. 